\title{
Influence of atomic kinetics in the simulation of plasma microscopic properties and thermal instabilities for radiative bow shock experiments
}

\author{
G. Espinosa, ${ }^{1,{ }^{*}}$ R. Rodríguez, ${ }^{1}$ J. M. Gil,${ }^{1}$ F. Suzuki-Vidal, ${ }^{2}$ S. V. Lebedev, ${ }^{2}$ A. Ciardi, ${ }^{3}$ J. G. Rubiano, ${ }^{1}$ and P. Martel ${ }^{1}$ \\ ${ }^{1}$ IUNAT, Departamento de Física, Universidad de Las Palmas de Gran Canaria, Spain \\ ${ }^{2}$ The Blackett Laboratory, Imperial College, London, United Kingdom \\ ${ }^{3}$ Sorbonne Université, UPMC Univ Paris 06, Observatoire de Paris, PSL Research University, CNRS, UMR 8112, \\ LERMA, F-75005, Paris, France
}

(Received 11 November 2016; revised manuscript received 31 January 2017; published 1 March 2017)

\begin{abstract}
Numerical simulations of laboratory astrophysics experiments on plasma flows require plasma microscopic properties that are obtained by means of an atomic kinetic model. This fact implies a careful choice of the most suitable model for the experiment under analysis. Otherwise, the calculations could lead to inaccurate results and inappropriate conclusions. First, a study of the validity of the local thermodynamic equilibrium in the calculation of the average ionization, mean radiative properties, and cooling times of argon plasmas in a range of plasma conditions of interest in laboratory astrophysics experiments on radiative shocks is performed in this work. In the second part, we have made an analysis of the influence of the atomic kinetic model used to calculate plasma microscopic properties of experiments carried out on MAGPIE on radiative bow shocks propagating in argon. The models considered were developed assuming both local and nonlocal thermodynamic equilibrium and, for the latter situation, we have considered in the kinetic model different effects such as external radiation field and plasma mixture. The microscopic properties studied were the average ionization, the charge state distributions, the monochromatic opacities and emissivities, the Planck mean opacity, and the radiative power loss. The microscopic study was made as a postprocess of a radiative-hydrodynamic simulation of the experiment. We have also performed a theoretical analysis of the influence of these atomic kinetic models in the criteria for the onset possibility of thermal instabilities due to radiative cooling in those experiments in which small structures were experimentally observed in the bow shock that could be due to this kind of instability.
\end{abstract}

DOI: 10.1103/PhysRevE.95.033201

\section{INTRODUCTION}

High-energy density (HED) laboratory plasma astrophysics is a research field whose popularity has grown considerably over the past three decades. It deals with the experimental modeling of astrophysical processes, involving studies of microphysics [1-3] and large-scale flow phenomena [4-6]. Two developments in the field have contributed to the successful design of HED laboratory astrophysical experiments. First, it was shown that magnetohydrodynamics can be scaled correctly between laboratory and astrophysical flows [7-12]. Second, the emergence of HED facilities, such as high-power lasers and fast magnetic pinch machines ( $\mathrm{Z}$ pinches), allow matter to be placed in extreme states of temperature, density, and velocity [9]. HED laboratory astrophysics includes, for example, experiments on radiative shocks [13-15], blast waves launched in atomic cluster media to emulate the ones observed in supernova remnants [16,17], and the formation of jets associated to newly forming stars [18-20]. Laboratory experiments have the advantage of being repeatable and that the initial conditions are under control and they also provide data for verification and validation of several aspects of numerical codes such as atomic physics, hydrodynamics, equation of state, and radiative transfer.

In many cases, the microscopic properties required in the radiative-hydrodynamics simulations of this kind of experiment (such as the average ionization, plasma level populations, or the radiative properties) are obtained with simplified population kinetic models, assuming, for example,

\footnotetext{
*guadalupe.espinosa@ulpgc.es
}

local thermodynamic equilibrium (LTE) for all the plasma conditions involved. However, this assumption could result in large errors for plasma conditions in which the plasma is in nonlocal thermodynamic equilibrium (NLTE) regime. Therefore, the analysis of the atomic kinetic model used to calculate the microscopic properties of the plasma will provide us which is the most appropriate at each plasma condition. Thus, for example, we could know if the radiative driven atomic processes due to external radiation fields are relevant, if plasma self-absorption should be considered in our kinetic model, if the steady-state assumption is accurate, or in which ranges of plasma conditions LTE approach could be assumed. These results also provide us a better understanding of the phenomena analyzed.

This work has been designed in this context and meets two objectives. The first one is the analysis of the validity of LTE assumption in the calculation of average microscopic properties of argon plasmas in mass densities and electron temperatures ranging from $10^{-6}$ to $10^{-1} \mathrm{~g} \mathrm{~cm}^{-3}$ and from 1 to $100 \mathrm{eV}$, respectively. Argon is an element commonly used in laboratory astrophysics experiments on radiative shocks generated using either pulsed power devices [19,21-24] or ultraintense lasers $[17,25-30]$, and the ranges of plasma conditions of these experiments fall within the ones before mentioned, hence, the interest of this study. For this analysis, we have made calculations assuming the plasma either in LTE and therefore using the Saha-Boltzmann (SB) equations, or in non-LTE (NLTE) in steady state, in which we have solved the rate equations implemented in our collisional-radiative model.

The second objective is to perform a study of the influence of the population kinetic models employed to calculate the plasma microscopic properties and to predict the possibility 
of thermal instabilities by radiative cooling in a particular HED laboratory astrophysics experiment where a radiative bow shock was generated by a radiatively cooled aluminum plasma jet in argon ambient gas [4]. Astrophysical jets are long, supersonic, and collimated outflows that can be observed emerging from a large variety of astrophysical objects, such as the nuclei of active galaxies, neutron stars, gamma ray bursts, or young stellar objects, among others [31]. The experiment analyzed in this work tries to reproduce jets from protostellar systems, the so-called Herbig-Haro objects. These jets are often the way to rid the system of its angular momentum, producing the possibility of a compact object formation. Sometimes, in these systems in formation, fine morphologies can be found like work surfaces at the head of these jets, and whose surface is normal to the flow direction. These structures are named bow shocks and their origin are the interaction with the surrounding medium. In the laboratory experiment under consideration in this work, the jet (see Suzuki-Vidal et al. [4]) was produced on the MAGPIE generator and was formed by ablation of an aluminum foil driven by a 1.4-MA, 250-ns current pulse in a radial foil Z-pinch configuration.

For this second objective, we have made calculations assuming the plasma either in LTE and in NLTE, but in the NLTE situation we have also analyzed the effect of an external radiation field (emitted by the radiatively cooled aluminum jet) in the bow shock and the effect of the presence of aluminum as an impurity in the argon plasma. The microscopic properties analyzed in the study have been the average ionization, the charge state distributions, the radiative power loss (RPL), the monochromatic emissivity and opacity, and the Planck mean opacity. The electron temperature and density of matter profiles used were obtained from a radiative-hydrodynamic simulation of the experiment using the magnetohydrodynamic code GORGON [32,33] in which LTE regime was assumed. We have also made an analysis of the influence of these atomic kinetic models in the analysis of thermal instabilities, which is a kind of instability of high interest in astrophysics since they can be involved in the generation of some astrophysics objects. All the study presented in this second objective was made as a postprocess of the hydrodynamic simulation. Detailed collisional-radiative models involve large computational costs and for this reason, integrated simulations, i.e. radiation-hydrodynamics simulations that calculate NLTE plasma properties in line [34], commonly use simpler atomic models than the one of this work and their accuracy could be limited. Therefore, we consider that the study carried out in this work based on the post-processing is pertinent. First, because the atomic model used is based on a relativistic detailed configuration accounting approach including many levels and transitions. And, second, because we have analyzed in this work not only the influence of NLTE, but also the importance of including other issues such as the external radiation field and the mixture of the argon plasma with aluminum in the calculation of plasma microscopic properties, which will further complicate an integrated simulation.

The remainder of the paper is organized as follows: In Sec. II we present the theoretical model used to calculate the microscopic properties of argon plasmas. Furthermore, in order to show the accuracy of this model, we made comparisons with calculations performed with other codes for some properties of argon plasmas. In Sec. III we perform the analysis of the validity of LTE approach in the calculation of mean radiative properties for argon plasmas in the range of plasma conditions typically found in laboratory experiments on radiative shocks. In Sec. IV we present the study of the influence of the plasma kinetics models in the numerical simulation of microscopic plasma properties for the particular experiment mentioned above. Furthermore, theoretical determination of the ranges of plasma conditions in the cooling region of the bow shock in which thermal instabilities could occur is addressed. In this section we also studied the influence of the atomic description, i.e., DCA or DLA approaches. Finally, in Sec. V the conclusions are presented.

\section{MODEL DESCRIPTION}

The simulation of microscopic properties such as the average ionization or the radiative properties of the argon plasma in the bow shock requires atomic data, such as energy levels and oscillator strengths as well as the atomic level populations at each plasma condition. In the following, the models employed in this work to calculate them are described.

\section{A. Atomic structure}

The atomic structure and the photoionization cross sections were calculated using the FAC code [35], in which a fully relativistic approach based on the Dirac equation is used. Hence, the atomic levels of an atomic ion are obtained by diagonalizing the relativistic Hamiltonian. The relativistic distorted wave approximation is used to calculate the photoionization cross section. The atomic data in FAC can be obtained either under the detailed configuration accounting (DCA) or detailed level accounting (DLA) approaches. For the former, the transition energies include the unresolved transition array (UTA) [36] shifts and a correction to the oscillator strengths due to the configuration interaction (CI) within the same nonrelativistic configurations. In the DLA approach, FAC can include different degrees of CI.

In this work, the atomic data were obtained in the DCA approach. Although this one is more accurate for high- $Z$ elements, we have checked that this approximation is accurate enough for the plasma properties analyzed. In any case, in Sec. IV C we present calculations of the radiative power loss calculated both in DCA and DLA approaches in order to show the effect of the atomic description used in the theoretical calculation of the criteria of thermal instabilities.

For the range of plasma conditions of interest in this work, with mass densities from $10^{-6}$ to $10^{-5} \mathrm{~g} \mathrm{~cm}^{-3}$ and electron temperatures between $10-15 \mathrm{eV}$, the ions of argon found are between $\mathrm{Ar}^{3+}$ and $\mathrm{Ar}^{10+}$. So, we have selected these ions to illustrate the atomic configurations included in this work in the DCA approach. For the former ion, the ground configuration is $[\mathrm{Ne}] 3 s^{2} 3 p^{3}$ and the configurations included were $3(s, p)^{m} 3 d^{m^{\prime}}$ (with $m=1-5, m^{\prime}=0-4, m+m^{\prime}=5$ ), where $(s, p)^{m}$ denotes all the configurations that arise from all the possible distributions of the $m$ electrons in subshells $3 s$ and $3 p$, $3(s, p)^{4}(n l)^{1}, 3(s, p)^{3} 3 d^{1}(n l)^{1}, 3(s, p)^{2} 3 d^{2}(n l)^{1} \quad$ (with $n=$ 4-10 and $l=0, \ldots, n-1), \quad 3(s, p)^{3} 4 l^{2}, 3(s, p)^{2} 3 d^{1} 4 l^{2}$, $3(s, p)^{3} 4 l^{1}\left(n^{\prime} l^{\prime}\right)^{1} \quad$ (with $l, l^{\prime}=s-f \quad$ and $\left.\quad n^{\prime}=5,6\right)$, 
$2(s, p)^{7} 3(s, p)^{6}, \quad$ and $2(s, p)^{7} 3(s, p)^{5} 3 d^{1}$. The ground configuration of $\mathrm{Ar}^{10+}$ is [He] $2 s^{2} 2 p^{4}$ and the configurations included in our simulations were $2(s, p)^{6}, 2(s, p)^{5}(n l)^{1}$ (with $n=3-10$ and $l=0, \ldots, n-1), 2(s, p)^{4}\left(n^{\prime} l\right)^{2}$ (with $\left.n^{\prime}=3,4\right), 2(s, p)^{4} 3 l^{1} 4 l^{\prime 1}$ (with $\left.l^{\prime}=s-f\right)$, and $2(s, p)^{3} 3 l^{3}$.

\section{B. Calculation of plasma atomic level populations}

Plasma level populations have been calculated in this work both in LTE and NLTE situations. For the former, the population of different ionization stages $N_{\zeta}$ can be obtained by solving the Saha equation

$$
\frac{N_{\zeta+1} n_{e}}{N_{\zeta}}=\frac{Z_{e} Z_{\zeta+1}}{Z_{\zeta}} e^{-\left(I_{\zeta}-\Delta I_{\zeta}\right) / k T_{e}},
$$

where $n_{e}$ is the free electron density, $Z_{e}$ and $Z_{\zeta}$ are the partition functions of free electrons and ion $\zeta$, respectively, $I_{\zeta}$ is the ionization potential of the ionization stage $\zeta$, and $\Delta I_{\zeta}$ is the depression of the ionization potential (continuum lowering, CL) due to the plasma environment, which can limit the number of bound states available in the plasma. We have used in this work for the CL the formulation developed by Stewart and Pyatt [37]. The Boltzmann distribution function is used to calculate the population of each atomic level belonging to the ionization stage $\zeta$.

In NLTE the atomic level populations are determined from the solution of a system of collisional-radiative (CR) rate equations. Our study in this work has been made under stationary state situations. Under this assumption, the kinetic rate equations of the $\mathrm{CR}$ steady-state (CRSS) model are given by

$$
\sum_{\zeta^{\prime} j} N_{\zeta^{\prime} j}(\mathbf{r}, t) R_{\zeta^{\prime} j \rightarrow \zeta i}^{+}-\sum_{\zeta^{\prime} j} N_{\zeta i}(\mathbf{r}, t) R_{\zeta i \rightarrow \zeta^{\prime} j}^{-}=0
$$

where $N_{\zeta i}$ is the population density of the atomic configuration or level $i$ (depending on the the atomic approach) of the ion with charge state $\zeta$. The terms $R_{\zeta^{\prime} j \rightarrow \zeta i}^{+}$and $R_{\zeta^{\prime} j \rightarrow \zeta i}^{-}$take into account all the atomic processes, both collisional and radiative, which contribute to populate and depopulate the state $\zeta i$, respectively. Two complementary equations have to be satisfied together with Eq. (2). First, the requirement that the sum of all the partial densities equals the total ion density $n_{\text {ion, }}$

$$
\sum_{\zeta=0}^{Z} \sum_{i=0}^{M_{\zeta}-1} N_{\zeta i}=n_{\mathrm{ion}},
$$

and, second, the charge neutrality condition in the plasma,

$$
\sum_{\zeta=0}^{Z} \sum_{i=0}^{M_{\zeta}-1} \zeta N_{\zeta i}=n_{e},
$$

where $M_{\zeta}$ is the total number of levels for the charge state $\zeta$. The plasma average ionization is defined as

$$
\bar{Z}=\frac{\sum_{\zeta=0}^{Z} \zeta N_{\zeta}}{\sum_{\zeta=0}^{Z} N_{\zeta}}=\frac{n_{e}}{n_{\text {ion }}} .
$$

The plasma charge state distribution (CSD) is defined as the set of the population densities $\left\{N_{\zeta}\right\}$ of the ions present in the plasma for a given condition of density and temperature. This is obtained from the resolution of either the rate (NLTE) or Saha-Boltzmann (LTE) equations.

The set of rate equations given by Eq. (2) would be coupled to the radiative transfer equation. However, in this work we have not considered the plasma self-absorption in the calculation of plasma level populations since we have checked that this effect is small. Therefore, the rate equations and the radiative transfer equation will be uncoupled. The atomic processes included in the CRSS are collisional ionization [38] and three-body recombination, spontaneous decay [35], collisional excitation [39] and deexcitation, radiative recombination [40], autoionization, and electron capture (obtained from the collisional excitation cross section using a known approximation [41]). The rates of the inverse processes are obtained through the detailed balance principle. Furthermore, since the bow shock is under the radiation field emitted by the radiatively cooled jet, we have also analyzed its influence in the microscopic properties of the plasma in the bow shock. In order to take into account the effect of external radiation fields in the calculation of the atomic level populations, the radiative driven processes of photoexcitation, photodeexcitation, and photoionization were included in the CRSS model. For the latter, the Kramers photoionization cross section [40] was used. The rates of these processes were obtained assuming that the intensity of the radiation field can be modeled with combinations of Planck functions.

In our CRSS model, multicomponent plasmas are also modeled. In this situation, collisional processes that connect ions of different chemical species in the plasma mixture were not included. Furthermore, since we are not considering plasma self-absorption effects in the CRSS model in this work, the coupling through the radiative transfer of the plasma self-radiation is not considered. On the other hand, as the different elements of the multicomponent plasmas are immersed into a common pool of free electrons, they will be coupled through the electron density since the average ionization of each component has to be consistent with the same electron density, which ensures the electrical equilibrium keeping the overall plasma neutrality $[42,43]$. Furthermore, the different components in the mixture are also coupled through the CL since in the formalism adopted in our CRSS model this one depends on the average ionization of the mixture. The coupling through the common free electron density and the CL takes into account the effect of the ionization competition between different atomic species on the population distribution and radiative properties [44] that might modify these properties compared with pure matters. For the experiment analyzed in this work, mixtures of $\mathrm{Ar}$ and $\mathrm{Al}$ in different proportions can be found and our procedure to perform the simulations of their microscopic properties was as follows: for a given density of matter and electron temperature of the profiles, provided by the radiative-hydrodynamic simulation using the GORGON code, we carried out an initial estimation of the total number electron density. Then, this density and the electron temperature were used to determine the rates for the individual rate matrices of $\mathrm{Ar}$ and $\mathrm{Al}$, and their rate equations were solved. The average ionizations obtained in conjunction with the relative abundances of $\mathrm{Ar}$ and $\mathrm{Al}$ and the density of matter were used to compute a new electron density for the mixture. 
This one was sent back to the single element population kinetics model for obtaining a new set of level populations and average ionizations. This process continued until the relative differences between all the fractional populations of each atomic level of both $\mathrm{Ar}$ and $\mathrm{Al}$ of two consecutive steps of the iterative procedure are lower than a certain tolerance imposed.

The atomic kinetic models used in this work to perform the numerical simulations of the radiative properties for monocomponent and multicomponent plasmas, both in LTE and NLTE regimes, are integrated in a numerical code named MIXKIP, an extension of a previous code ABAKO [45,46], developed to model steady- and non-steady-state multicomponent plasmas.

\section{Calculation of the plasma radiative properties}

Plasma radiative properties are calculated using the RAPCAL code $[46,47]$. The monochromatic emissivity and absorption are denoted in this work as $j(v)$ and $\kappa(v)$, respectively (where we have omitted the dependence on the position and time to simplify the notation) and both of them include the boundbound, bound-free, and free-free contributions

$$
\begin{gathered}
j(v)=j_{b b}(v)+j_{b f}(v)+j_{f f}(v), \\
\kappa(v)=\kappa_{b b}(v)+\kappa_{b f}(v)+\kappa_{f f}(v),
\end{gathered}
$$

where $v$ is the photon frequency. The bound-bound contribution to the emissivity is given by

$$
j_{b b}(v)=\sum_{\zeta} \sum_{i, j} j_{\zeta j \rightarrow \zeta i}(v),
$$

with

$$
j_{\zeta j \rightarrow \zeta i}(v)=\frac{h v}{4 \pi} N_{\zeta j} A_{\zeta j \rightarrow \zeta i} \phi_{i j}(v),
$$

where $A_{\zeta j \rightarrow \zeta i}$ is the Einstein coefficient for spontaneous deexcitation between the bound states $j, i$ of the ion $\zeta$ and $h$ is the Planck's constant. The radiative transitions rates in FAC are calculated in the single multipole approximation, and in this work they were obtained in the electric dipole approach. The bound-bound contribution to the absorption is given by

$$
\kappa_{b b}(\nu)=\sum_{\zeta} \sum_{i, j} \kappa_{\zeta i \rightarrow \zeta j}(v),
$$

with

$\kappa_{\zeta i \rightarrow \zeta j}(v)=\frac{h v}{4 \pi} N_{\zeta i} \frac{g_{\zeta j}}{g_{\zeta i}} \frac{c^{2}}{2 h v_{i j}^{3}} A_{\zeta j \rightarrow \zeta i} \phi_{i j}(v)\left(1-\frac{g_{\zeta i}}{g_{\zeta j}} \frac{N_{\zeta j}}{N_{\zeta i}}\right)$

where $c$ is the speed of light and $g_{\zeta i}$ and $g_{\zeta j}$ are the statistical weights of the $i$ and $j$ levels. In the previous equations, $\phi_{i j}(v)$ represents the line profile both for line emission and absorption since in this work complete redistribution hypothesis is assumed [48]. In the evaluation of the line profile, natural, Doppler, and electron-impact [49] broadenings were included and also the UTA width in the DCA approach. The line-shape function is applied with the Voigt profile that incorporates all these broadenings.
The bound-free contribution to the emissivity is determined by means of

$$
j_{b f}(v)=\sum_{\zeta, i} \sum_{\zeta, j} j_{\zeta+1 j \rightarrow \zeta i}(v)
$$

with

$$
\begin{aligned}
& j_{\zeta+1 j \rightarrow \zeta i}(v) \\
& \quad=\frac{h}{2 \pi c^{2}}\left(\frac{1}{2 m_{e}}\right)^{3 / 2} N_{\zeta+1 j} n_{e} f(\varepsilon) \frac{g_{\zeta i}}{g_{\zeta+1 j}} \frac{(h v)^{3}}{\varepsilon^{1 / 2}} \sigma_{\zeta i \rightarrow \zeta+1 j}^{\text {pho }}(v),
\end{aligned}
$$

with $\varepsilon$ the energy of the free electron and $m_{e}$ the electron mass. In this work, a Maxwell-Boltzmann distribution $f(\varepsilon)$ at temperature $T_{e}$ for the free electrons is assumed. Photoionization cross section $\sigma_{\zeta i \rightarrow \zeta+1 j}^{\text {pho }}(v)$ has been calculated using FAC code in the relativistic distorted wave approach. For the bound-free spectra, the DCA approximation has been used as well. The bound-free contribution to the absorption is given by

$$
\kappa_{b f}(v)=\sum_{\zeta, i} \sum_{\zeta, j} \kappa_{\zeta i \rightarrow \zeta+1 j}(\nu),
$$

with

$\kappa_{\zeta i \rightarrow \zeta+1 j}(v)=N_{\zeta i} \sigma_{\zeta i \rightarrow \zeta+1 j}^{\text {pho }}(v)\left[1-\frac{N_{\zeta+1 j} n_{e} f(\varepsilon) g_{\zeta i}}{N_{\zeta i} g_{\zeta+1 j} g(\varepsilon)}\right]$,

where $g(\varepsilon)$ is the density of states with energy $\varepsilon$ which, assuming an ideal gas of free electrons, is given by

$$
g(\varepsilon)=4 \pi\left(\frac{2 m_{e}}{h^{2}}\right)^{3 / 2} \varepsilon^{1 / 2} .
$$

For the free-free contributions to the emissivity and the absorption, the Kramers semiclassical expression for the inverse bremsstrahlung cross section has been used [50]:

$$
\sigma_{\zeta}^{i b r}(v)=\frac{16 \pi^{2} e^{2} h^{2} \alpha}{3 \sqrt{3}\left(2 \pi m_{e}\right)^{3 / 2}} \frac{\zeta^{2} n_{e}}{T_{e}^{1 / 2}(h v)^{3}} .
$$

Assuming a Maxwell-Boltzmann distribution for the free electrons, we obtain

$$
\begin{gathered}
j_{f f}(\nu)=\frac{32 \pi^{2} e^{4} a_{0}^{2} \alpha^{3}}{\sqrt{3}\left(2 \pi m_{e}\right)^{3 / 2} h}\left(\frac{m_{e}}{2 \pi T_{e}}\right)^{1 / 2} \overline{Z^{2}} n_{\text {ion }} n_{e} e^{-h \nu / T_{e}}, \\
\kappa_{f f}(\nu)=\frac{16 \pi^{2} e^{2} h^{2} \alpha}{3 \sqrt{3}\left(2 \pi m_{e}\right)^{3 / 2}} \frac{\overline{Z^{2}} n_{\text {ion }} n_{e}}{T_{e}^{1 / 2}(h \nu)^{3}}\left(1-e^{-h \nu / T_{e}}\right) .
\end{gathered}
$$

In order to determine the opacity $k(v)$, the absorption due to the scattering of photons is also taken into account. In RAPCAL this one is approximated using the Thomson scattering cross section [51]

$$
\kappa_{\text {scatt }}=n_{e} \sigma^{\text {Thom }},
$$

with $\sigma^{\text {Thom }}=6.65 \times 10^{-25} \mathrm{~cm}^{2}$.

Finally, the opacity is given by

$$
k(v)=\frac{1}{\rho}\left[\left(\kappa(v)+\kappa_{\text {scatt }}\right],\right.
$$

with $\rho$ the density of matter. As said before, RAPCAL also provides the Planck $k_{P}$ and Rosseland $k_{R}$ mean opacities, 
which are given by [52]

$$
\begin{gathered}
k_{P}=\int_{0}^{\infty} d \nu \tilde{B}(\nu, T)\left[k(\nu)-\kappa_{\mathrm{scatt}} / \rho\right], \\
\frac{1}{k_{R}}=\int_{0}^{\infty} d \nu \frac{\partial \tilde{B}(\nu, T)}{\partial T} \frac{1}{k(\nu)},
\end{gathered}
$$

where $\tilde{B}(\nu, T)$ is the normalized Planckian function

$$
\tilde{B}(v, T)=\frac{15}{\pi^{4} T} \frac{u^{3}}{e^{u}-1}, \quad u=\frac{h v}{T} .
$$

The radiative power loss (RPL) is evaluated as

$$
P=4 \pi \int_{0}^{\infty} j(v) d v .
$$

\section{Comparisons with other models}

We present comparisons of some plasma properties, average ionization, CDS and RPL, for optically thin argon in density and temperature conditions of interest in this work in order to show the accuracy of our simulations in these conditions. The comparisons are made with calculations performed by NLTE kinetic codes that participated in the Fifth Non-LTE Code Comparison Workshop [53].

Table I shows a comparison of the average ionization for a plasma of argon at six electron temperatures and two electron densities. In general, the relative differences obtained with respect to the other codes are lower than $10 \%$. Both the set of atomic configurations considered and the atomic description in each code are different and, therefore, these differences are expected. For the case of lowest temperature and highest density presented in the table, the largest differences $(>20 \%)$ are obtained. This could be due to the model of CL implemented in each code since this effect becomes more relevant as the density increases and the temperature decreases. On the

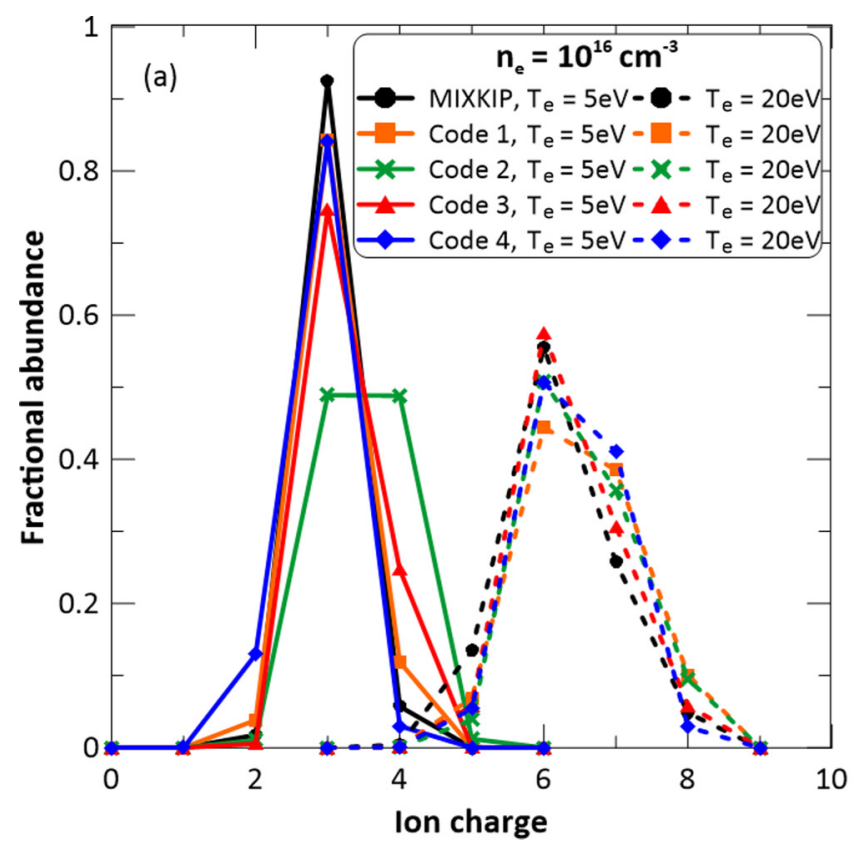

TABLE I. Comparison of the average ionization, at several electron temperatures and two electron densities, with calculations of codes that participated in the Fifth Non-LTE Code Comparison Workshop [53].

\begin{tabular}{lcccccc}
\hline \hline$T_{e}(\mathrm{eV})$ & $n_{e}\left(\mathrm{~cm}^{-3}\right)$ & MIXKIP & Code 1 & Code 2 & Code 3 & Code 4 \\
\hline 3 & $10^{16}$ & 2.229 & 2.071 & 2.200 & 2.272 & 1.954 \\
& $10^{20}$ & 0.417 & 0.319 & 1.051 & 0.584 & 0.673 \\
5 & $10^{16}$ & 3.040 & 3.081 & 3.502 & 3.243 & 2.900 \\
& $10^{20}$ & 1.823 & 1.583 & 1.981 & 1.593 & 1.674 \\
8 & $10^{16}$ & 3.894 & 4.053 & 4.810 & 4.430 & 3.571 \\
& $10^{20}$ & 3.194 & 3.200 & 3.237 & 2.938 & 2.685 \\
13 & $10^{16}$ & 4.982 & 5.189 & 5.640 & 5.620 & 5.196 \\
& $10^{20}$ & 5.219 & 5.234 & 5.118 & 4.947 & 5.222 \\
20 & $10^{16}$ & 6.211 & 6.515 & 6.509 & 6.365 & 6.412 \\
& $10^{20}$ & 7.105 & 7.122 & 7.037 & 6.581 & 6.599 \\
35 & $10^{16}$ & 7.822 & 7.846 & 7.835 & 7.646 & 7.099 \\
& $10^{20}$ & 7.911 & 7.992 & 8.007 & 8.001 & 7.931 \\
\hline \hline
\end{tabular}

other hand, the better agreement among all the codes is found for the situation of lowest density and highest temperature. In this case, the average ionization is around 8, which means that the most abundant ion is the Ne-like one. This ion has an atomic structure of closed shell and therefore the set of atomic configurations is simpler than when the ion has open shells. Therefore, the codes would include similar atomic configurations in their simulations which could justify the better agreement found in this case. Furthermore, the effect of the CL for this density is not significant, which also contributes to the agreement. Figure 1 complements information presented in Table I, where we have represented a comparison of the CSDs at two electron temperatures $(5$ and $20 \mathrm{eV}$ ) for the two electron densities considered in this section. We can observe that our model provides CSDs similar to the ones obtained with

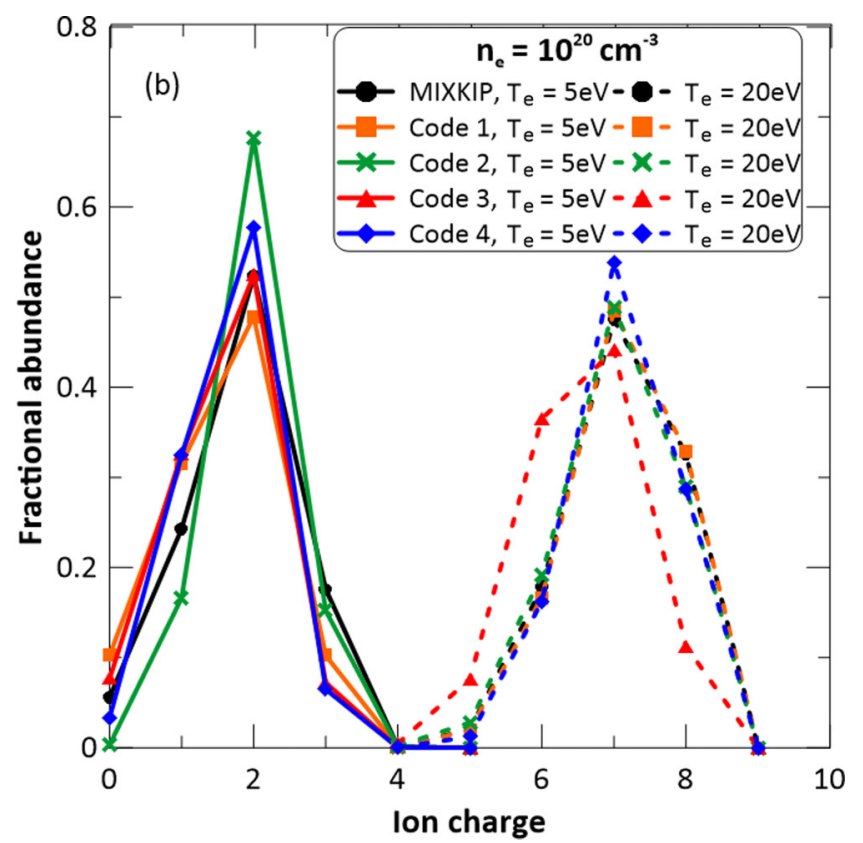

FIG. 1. Comparison of the charge state distributions at two electron temperatures and densities with calculations of codes that participated in the Fifth Non-LTE Code Comparison Workshop [53]. 

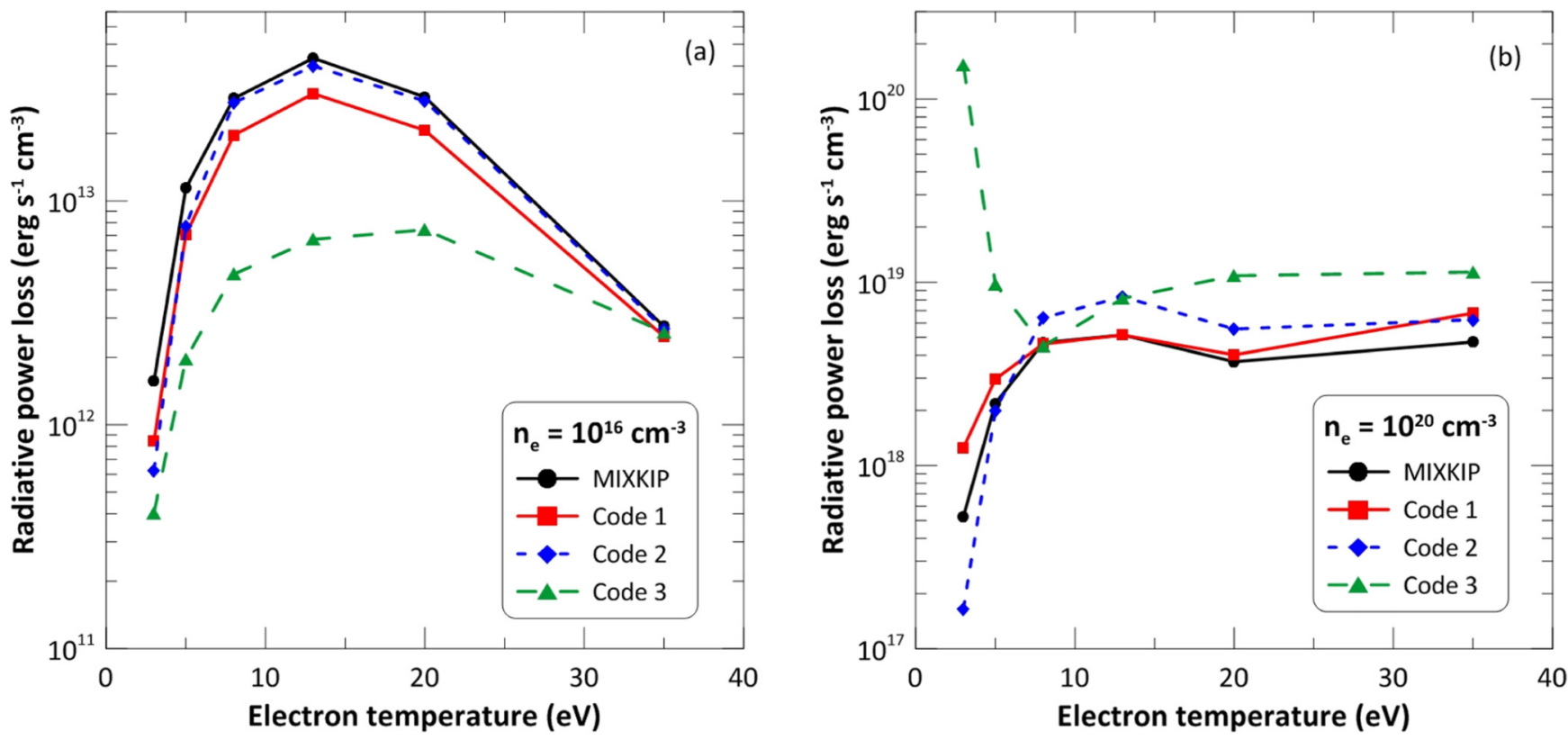

FIG. 2. Comparison of the radiative power loss at several electron temperatures and two electron densities, with calculations of codes that participated in the Fifth Non-LTE Code Comparison Workshop [53].

the other codes. As we detected for the average ionization, the agreement among the codes gets worse as the density increases and the temperature decreases. Thus, for example, at $5 \mathrm{eV}$ and $10^{20} \mathrm{~cm}^{-3}$, we can observe in the figure that although the most abundant ions predicted by all the codes are the same, the fractional abundances change appreciably.

Finally, in Fig. 2 we present a comparison of the RPLs calculated with MIXKIP with the ones provided by the other codes of the workshop. The plasma conditions are the same as for the average ionization and the CSDs. Although the RPL is also an averaged property (in photon frequencies), it shows a stronger dependence both on the atomic structure and on the plasma ion level populations than the other two properties analyzed, and, therefore, greater differences between code simulations could be expected. From the figure, we observe that our simulations and the ones obtained using codes 1 and 2 are quite similar for nearly all the plasma conditions. However, the results obtained using code 3 show great differences and even a different behavior with the temperature for the highest mass density case. As expected, the better agreement among the codes is obtained at the lowest density and highest temperature considered. Therefore, the whole set of comparisons presented for argon in plasma conditions which are of interest in this work give us confidence that our atomic and population kinetic models are accurate enough for the analysis of the microscopic properties that we carried out in this work.

\section{STUDY OF THE THERMODYNAMIC REGIME OF ARGON PLASMAS}

As said before, Ar is an element frequently used in astrophysics experiments on radiative shocks. In most of them, the mass densities and electron temperatures are ranged from $10^{-6}$ to $-10^{-1} \mathrm{~g} \mathrm{~cm}^{-3}$ and from 1 to $100 \mathrm{eV}$, respectively. In this section, we have made a study of the influence of the thermodynamic regime in the calculation of averaged microscopic properties of interest such as the average ionization, the radiative power loss, the Planck and Rosseland mean opacities, and the cooling time in that range of plasma conditions. For that purpose, we have compared the results obtained using the $\mathrm{SB}$ and the rate equations of the CRSS model implemented in MIXKIP.

Figure 3 displays the comparison of LTE and NLTE simulations of the average ionization, Planck and Rosseland mean opacities, and RPL for the whole range of temperatures considered and for four cases of mass densities, including the lowest and the greatest ones. From the comparison we detect that for the greatest density considered $\left(10^{-1} \mathrm{~g} \mathrm{~cm}^{-3}\right)$, the agreement in the average ionization between both simulations is excellent with relative differences always lower than $2 \%$ in the whole range of temperatures. The agreement is also good for the mean opacities and the RPL with relative differences between both simulations, in general, lower than $5 \%$. The relative differences in these properties are larger than for the average ionization since they are more sensitive to changes in the plasma level populations than the latter. In any case, we can conclude that for the density of $10^{-1} \mathrm{~g} \mathrm{~cm}^{-3}$ the LTE approach is accurate enough to calculate these average plasma properties.

For the density of matter of $10^{2} \mathrm{~g} \mathrm{~cm}^{-3}$, the relative differences in the average ionization are lesser than $5 \%$ for temperatures up to $60 \mathrm{eV}$, reaching $10 \%$ for higher temperatures, since for a fixed density of matter as the temperature increases the departure of the plasma from the LTE regime is larger. With respect to the mean radiative properties relative differences, we have obtained relative differences lower than $20 \%$ for temperatures up to $50 \mathrm{eV}$ and around $30 \%$ for higher temperatures. Therefore, for this density case although the relative differences between both models for the average ionization are not too large, we begin to detect appreciable 

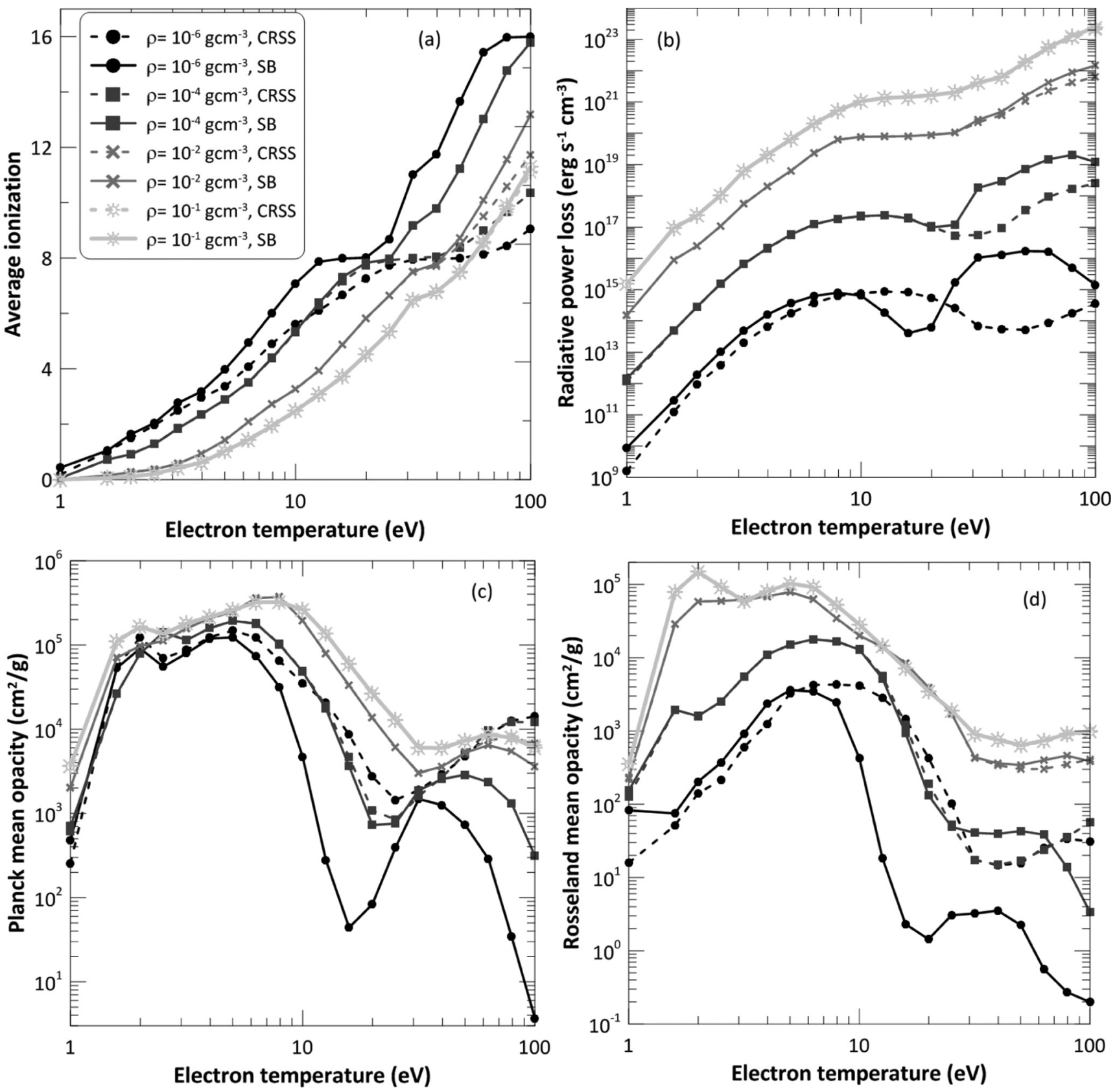

FIG. 3. Comparison of the average ionization (a), the RPL (b), and the Planck (c) and Rosseland (d) mean opacities obtained using SB and CRSS models. The legend shown in (a) is valid for all the figures.

differences in the radiative properties. These differences become significantly larger as the mass density decreases. Thus, the relative differences in the average ionization between LTE and NLTE simulations can reach $50 \%$ and $90 \%$ for the mass densities of $10^{-4}$ and $10^{-6} \mathrm{~g} \mathrm{~cm}^{-3}$, respectively, for temperatures higher than $70 \mathrm{eV}$. The disagreement is even worse for the mean radiative properties, obtaining for instance relative differences of $100 \%$ in almost the whole range of temperatures for the density of $10^{-6} \mathrm{~g} \mathrm{~cm}^{-3}$. Therefore, it is clear that for these two mass densities the plasma is far from LTE.

We have also analyzed the influence of the plasma thermodynamic regime in the calculation of a relevant parameter in the analysis of radiative shocks that is related with the plasma properties already studied. This is the cooling time and is given by (in s)

$$
t_{\mathrm{cool}}=2.42 \times 10^{-12} \frac{(\bar{Z}+1) n_{\mathrm{ion}} T_{e}}{\left|\nabla \cdot \vec{F}_{\mathrm{rad}}\right|},
$$

where $\vec{F}_{\text {rad }}$ is the radiative flux and its divergence (given in $\operatorname{erg} \mathrm{cm}^{-3} \mathrm{~s}^{-1}$ in the previous equation), assuming that the radiation does not depend explicitly on time, is given by

$$
-\nabla \cdot \vec{F}_{\text {rad }}=4 \pi \int_{0}^{\infty} j(v) d v-4 \pi \int_{0}^{\infty} \kappa(v) J(v) d v,
$$

where $J(v)$ is the mean spectral intensity. In the previous equation we have omitted for simplicity the dependence of 


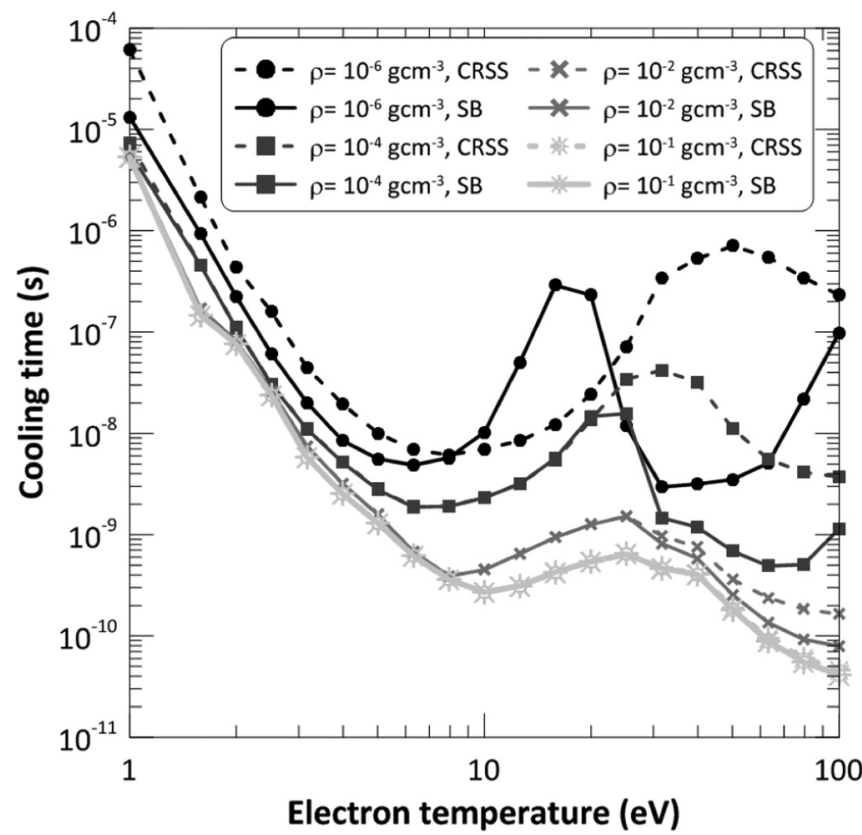

FIG. 4. Comparison of the cooling times obtained using SB and CRSS models.

the radiative properties on time, position, and propagation direction. The importance of the cooling time in the analysis of radiative shocks will be commented in the next section. Here, we will restrict ourselves to make a comparison of the cooling times obtained using the CRSS and SB models. We will assume the plasma as optically thin, and then, from Eq. (27), the divergence of the radiative flux is reduced to the RPL. Therefore, the cooling time depends on the average ionization and the RPL and all the conclusions obtained in the analysis of these two properties remain valid. Thus, the agreement in the cooling times obtained with both models is quite good for the two highest densities shown in Fig. 4. On the other hand, noticeable discrepancies are detected for the other two mass densities represented and even different behaviors with temperature are obtained. Since the cooling time is related to the radiative character of the shock and with the possibility of thermal instabilities, as will be discussed in next section, these differences between both models could be relevant.

\section{ANALYSIS OF AN EXPERIMENTAL RADIATIVE BOW SHOCK}

\section{A. Experimental setup}

In this section, we present a brief description of the experiment that we have analyzed in this work. We mainly focused our attention here in those aspects which are more related with the study we have carried out. An extensive description of the experiment can be found in Ref. [4]. Using the MAGPIE power pulsed device, supersonic radiatively cooled jets were produced by the ablation of plasma from surface of aluminum foil driven by the 1.4-MA, 250-ns current pulse in a radial foil Z-pinch configuration. Argon gas was introduced into the region above the foil in order to investigate the jet-ambient gas interaction. The addition of argon affects the formation and evolution of the jet with respect to the situation in which the jets propagated in vacuum. The presence of argon does not disrupt the formation of the jet but produces the appearance of some new features. One of them is a shock feature propagating on the axis of the jet and centered on its tip, which is the bow shock. The extreme ultraviolet (XUV) images obtained in the experiment show that the bow shock appears around $300 \mathrm{~ns}$. This one starts with a quasispherical shape and expands both axially and radially [4] [see Fig. 5(a)]. The images also show that the XUV emission in the front of the bow shock is almost as strong as at the tip of the jet and therefore could be considered as a radiative shock. Furthermore, a Schlieren diagnostic of the bow shock also revealed the presence of small-scale structures $(100 \mu \mathrm{m})$ forming in the shock, that could be related to thermal instabilities due to radiative cooling. Numerical simulations of the experiment were performed using the GORGON code [33] [an example can be seen in Fig. 5(b)] which is an explicit, parallel code designed to solve the resistive MHD equations on a three-dimensional Cartesian grid. The axial profiles at 300 and $400 \mathrm{~ns}$ of density of matter and electron temperature for the bow shock provided by GORGON [the axial profiles at $300 \mathrm{~ns}$ are represented as example in Fig. 5(c)] have been used in this work in order to perform the collisional-radiative simulations. In GORGON, LTE was assumed to calculate the ionization degrees.

\section{B. Collisional-radiative simulation of the argon plasma in the bow shock}

From the numerical simulations of GORGON it was obtained that the mass densities and electron temperatures of the plasma
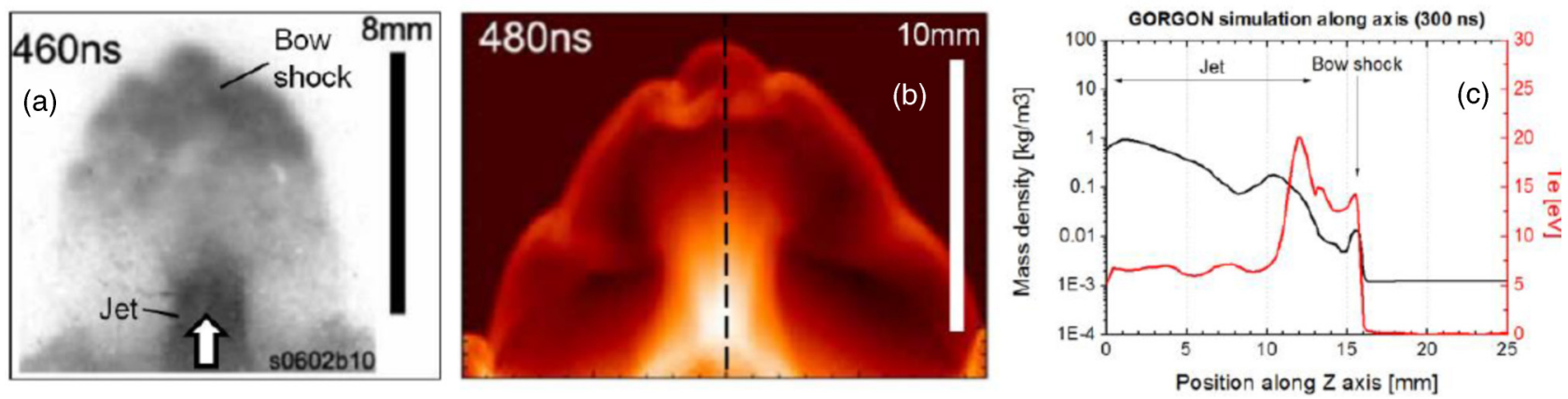

FIG. 5. Formation of a bow shock from the interaction of an aluminum jet with an argon ambient. (a) Experimental results on the MAGPIEgenerator (XUV emission); (b) numerical simulations of the experiment with the code GORGON; (c) profiles of mass density and electron temperature from simulations on axis at $300 \mathrm{~ns}$. 


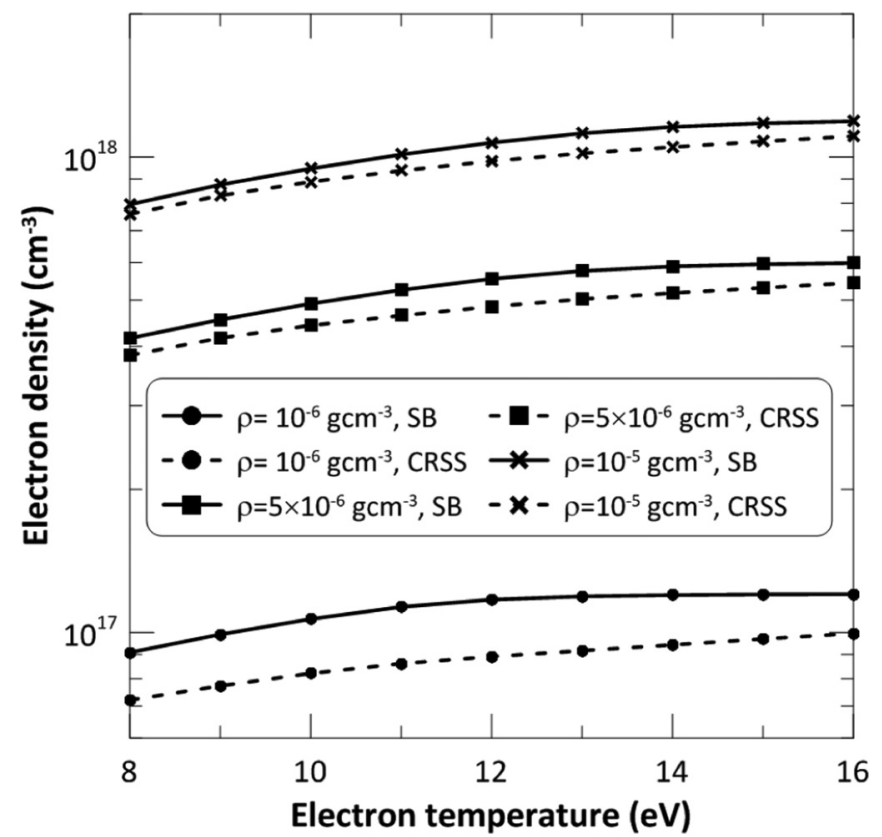

FIG. 6. Electron densities in terms of the electron temperatures for the range of mass densities of the experiment, calculated using SB and CRSS models.

in the bow shock were ranged from $10^{-6}$ to $10^{-5} \mathrm{~cm}^{-3}$ and from 10 to $15 \mathrm{eV}$, respectively. In Fig. 6, we have represented the electron densities as a function of the electron temperature corresponding to this range of mass densities calculated using both SB and CRSS models. From the figure we can observe that they are ranged from $7 \times 10^{16}$ to $10^{18} \mathrm{~cm}^{-3}$.

For the study performed in this work we made a $Z$-axis discretization of the plasma in the bow shock dividing it into cells of homogeneous density and temperature conditions of varying lengths. The microscopic properties analyzed at each cell were the average ionization, the CSDs, the monochromatic opacities and emissivities, the Planck mean opacities, and the radiative power losses. For their calculation both LTE (this approach was assumed in the hydrodynamic simulation) and NLTE population kinetic models were used, solving SahaBoltzmann and rate equations, respectively. Furthermore, for the NLTE assumption we have made several different simulations assuming a pure argon plasma and a plasma mixture of argon and aluminum and including or not the axial radiation coming from the jet as an external radiation field. The rates of the radiative processes induced by that radiation are modeled in MIXKIP as a combination of Planckian functions with their corresponding radiation temperature and dilution factor. Figure 7 illustrates this modeling. In the figure we present the intensities of the radiation emitted by the jet in the axial direction at two times, which we had already simulated and studied in a previous paper [54], and their approximation by two Planckian functions. The factors that multiply the Planckian functions do not have geometric or physics meaning, they are obtained from the numerical fitting to the specific intensities. The intensities were obtained assuming either a pure aluminum jet or a plasma mixture in the jet of $50 \%$ of argon and aluminum since, according to Suzuki-Vidal et al. [4], argon can be trapped inside the body of the jet during the early stages of its formation and its concentration could reach the $50 \%$ in the axial region of the jet. The first thing that we can observe from the figures is that the combination of the two Planck's functions acceptably reproduces the specific intensities. The fitting was made in the photon energy range 1-200 eV since we have checked that the bow shock presents the main absorption peaks in that range and the intensity of the radiation coming from the jet is greater (see Fig. 7). On the
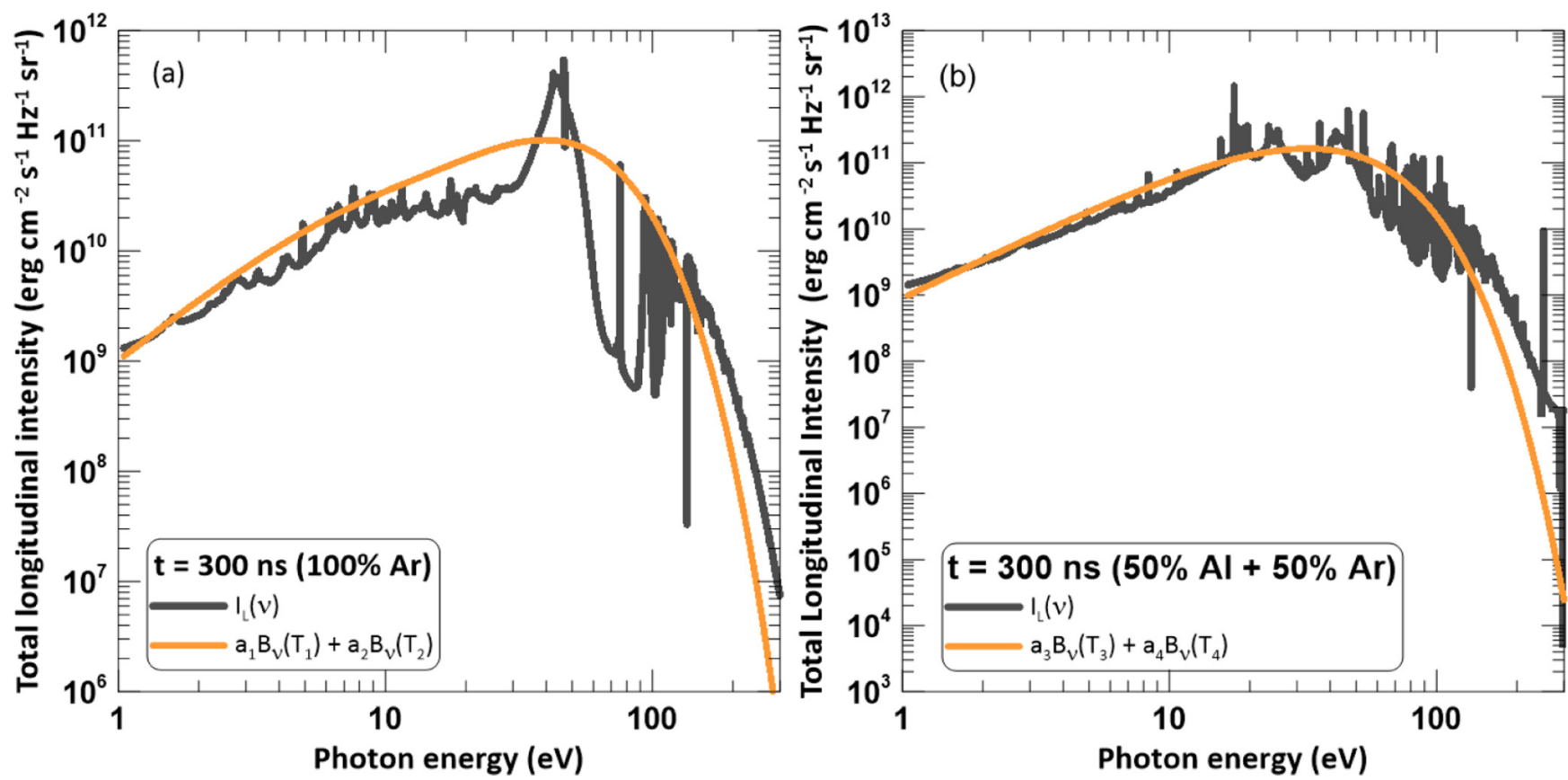

FIG. 7. Fitting of the intensity emitted by the jet in the axial direction using two Planckian functions. (a) Assuming the jet with Al only. $T_{1}=3 \mathrm{eV}$ and $T_{2}=14 \mathrm{eV}, a_{1}=2$ and $a_{2}=0.2$. (b) Assuming a plasma mixture in the jet of $50 \% \mathrm{Al}$ and $50 \% \mathrm{Ar}$. $T_{3}=6 \mathrm{eV}$ and $T_{4}=13 \mathrm{eV}$, $a_{3}=0.2$ and $a_{4}=0.5$. 

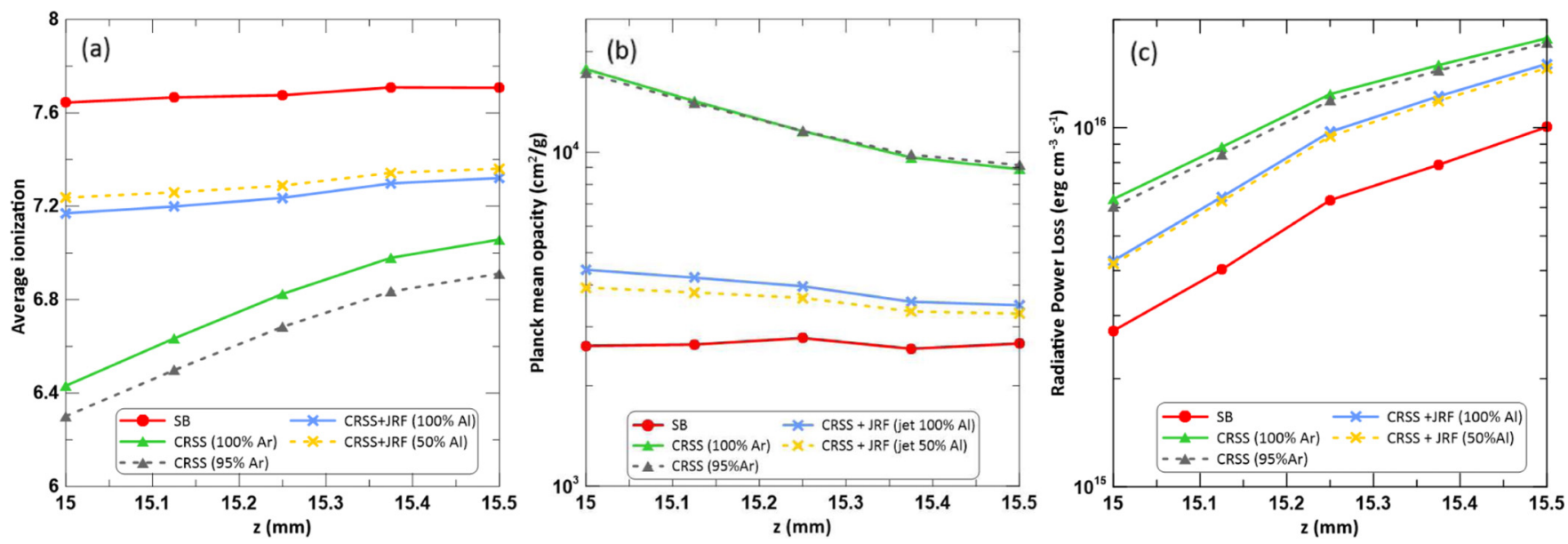

FIG. 8. Comparison of the (a) average ionization, (b) Planck mean opacity, and (c) radiative power loss, calculated with the different population kinetics models as a function of the $z$ coordinate at $300 \mathrm{~ns}$.

other hand, we can also detect that the radiation temperatures of the Planckian functions change when argon is included in the jet. This is due to the fact that the specific intensity of the mixture presents new features with respect to the situation of pure aluminum jet, as for example at $300 \mathrm{~ns}$ the emission peaks in the photon energy ranges $10-30 \mathrm{eV}$ and $60-90 \mathrm{eV}$ increasing the specific intensities in those ranges.

In Fig. 8, we have represented the behavior of the average ionization, the Planck mean opacity, and the RPL calculated with the different population kinetics models at $300 \mathrm{~ns}$ as a function of the axial coordinate. From the profiles given by the hydrodynamic simulation [see Fig. 5(c)] we have extracted that the front shock is located at $z \approx 15.5 \mathrm{~mm}$ and the post shock ends at $z \approx 15.0 \mathrm{~mm}$.

As expected, we detect in the average ionization that the values provided by the SB model, i.e., assuming LTE, are always larger than the ones obtained with the CRSS models since SB equations overestimate the average ionization when the plasma is out of the LTE. On the other hand, the lowest values for the average ionization are the ones calculated with the CRSS models without including the radiation field of the jet. The relative differences between both models are ranged from $16 \%$ to $8.5 \%$, decreasing the differences as the density increases, i.e., as the $z$ coordinate increases, and then closer to the shock front since this fact encourages to achieve the LTE regime.

We also observe that the inclusion of some $\mathrm{Al}(5 \%)$ into the simulation [CRSS (95\% Ar) in the figure] leads to a little reduction in the ionization (around $2 \%$ ). We have detected in the range of plasma conditions analyzed the average ionization of $\mathrm{Al}$ (around 4) is lower than the one for Ar (around 6-7) and this fact produces the reduction of the average ion in the mixture. Besides, $\mathrm{Al}^{3+}$ is a relevant ion for these plasma conditions but it has Ne-like atomic structure (closed shell) and higher temperatures than the ones of the range under consideration are required to increase the $\mathrm{Al}$ ionization.

When the radiation field emitted by the jet is included in the CRSS model [CRSS+JRF (jet radiation field) models in the figure] we detect an increase of the average ionization, with relative differences between $11.5 \%$ and $3.7 \%$, which means that the radiative processes induced by the external radiation field influence in the plasma ionization. Again, as the density increases, the differences diminish what it is expected since the contribution of the collisional processes becomes more important. The increase of the ionization is slightly greater when the radiation of the jet is modeled assuming this as a mixture of $\mathrm{Al}$ and $\mathrm{Ar}$ [at $50 \%$ each one of them, denoted as CRSS+JRF $(50 \% \mathrm{Al})$ in the figure] which could be due to a higher emission from Ar than from $\mathrm{Al}$ as can be seen in Fig. 7 , where some of the valleys of the specific intensities of the radiation emitted by $\mathrm{Al}$ are filled in due to the emission of Ar. However, the relative differences between the two models that include the radiation field are small (around 1\%).

In Fig. 9, we have displayed an example of the CSDs, that complements the analysis of the average ionization, at $300 \mathrm{~ns}$ for $z=15.0$ and $15.5 \mathrm{~mm}$, which are the positions in which the mass density reaches its maximum and minimum values $5 \times$ $10^{-6}$ and $1.5 \times 10^{-5} \mathrm{~g} \mathrm{~cm}^{-3}$, respectively, at that time. The electron temperatures are 13 and $14.5 \mathrm{eV}$, respectively. From the figure, we observe that in both positions the most abundant ions in the SB model are $\mathrm{Ar}^{7+}$ and $\mathrm{Ar}^{8+}$. The latter ion has the electronic structure of $\mathrm{Ne}$ and therefore larger changes in the temperature would need to increase the average ionization and then this one remains almost constant (see Fig. 8). For the CRSS models without including the jet radiation the most abundant ions are $\mathrm{Ar}^{6+}$ and $\mathrm{Ar}^{7+}$ at $z=15.0$ increasing the abundance of $\mathrm{Ar}^{7+}$ and $\mathrm{Ar}^{8+}$ ions at $z=15.5$ mainly due to the rise of the electron temperature. As for the average ionization, the effect in the CSD of the Al and Ar mixture is not significant, as we can see in the figure. As commented before, the radiation field causes an increase in the ionization, which leads to higher abundances of the $\mathrm{Ar}^{7+}$ and $\mathrm{Ar}^{8+}$ ions with respect to the situation without external radiation in both axial positions. However, as in the case of the SB model, the changes in the fractional abundances with temperature are lower than in the CRSS model without including the external radiation field, due to the presence of the $\mathrm{Ar}^{8+}$ ion.

In Fig. 8, we have also displayed the comparisons for the Planck mean opacity and the RPL. The relative differences between the results provided by the different models are larger than for the average ionization. This fact is expected since both properties are more sensitive to the changes in ion and 

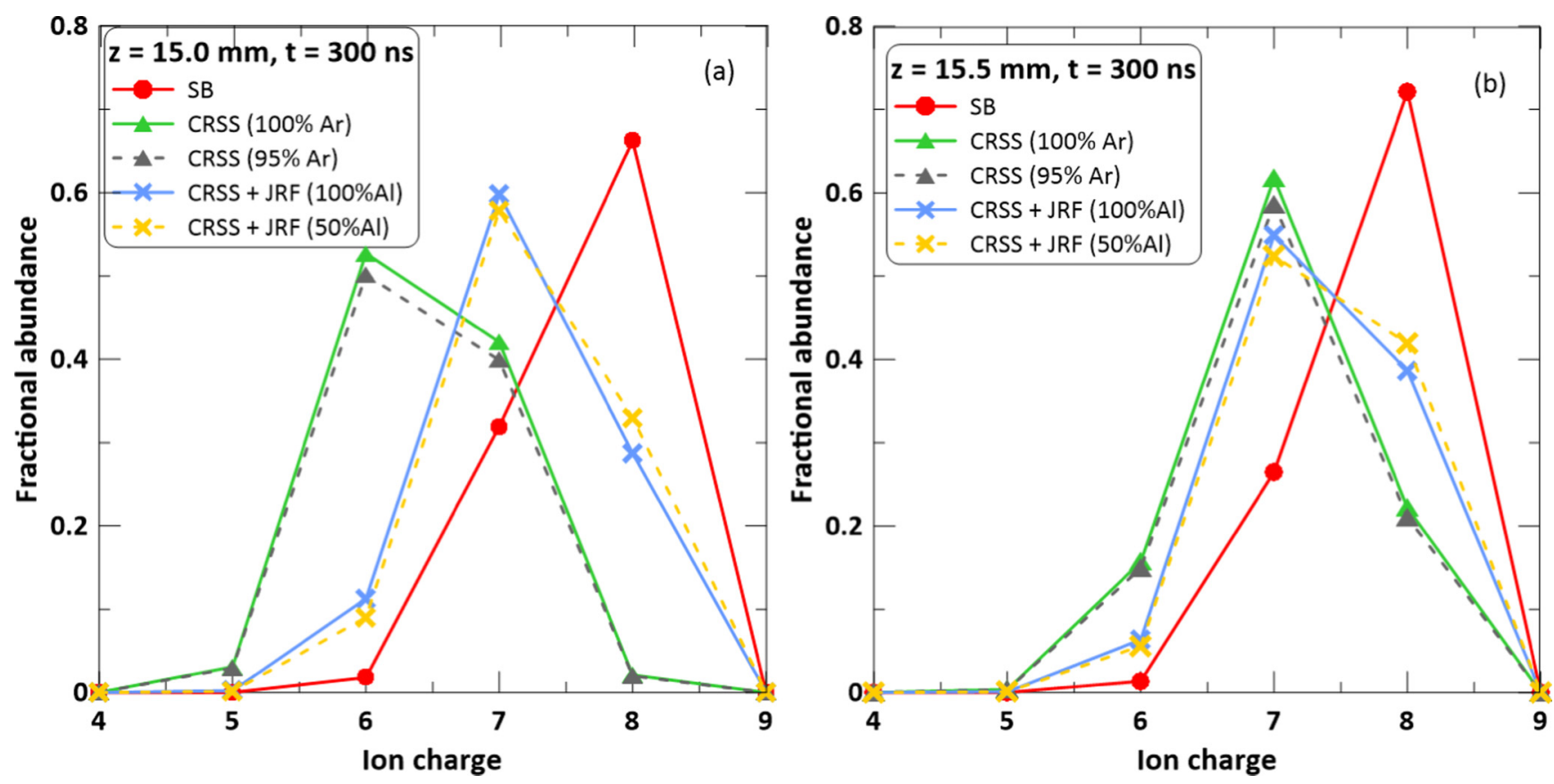

FIG. 9. Comparison of the CSD calculated with the different population kinetics models at $300 \mathrm{~ns}$ for two values of the $z$ coordinate.

plasma level populations than the average ionization. Thus, for example, the relative differences in the Planck opacity and the RPL between the CRSS model assuming a pure Ar bow shock without including external radiation fields and the SB model ranges between $85 \%-70 \%$ and $55 \%-45 \%$, respectively, and between that CRSS model and the one including the radiation field assuming a pure aluminum jet are $75 \%-60 \%$ and $30 \%-15 \%$, respectively. On the other hand, the relative differences between the two models that include the external radiation field and between the CRSS models considering the bow shock including or not $\mathrm{Al}$ remain small, such as in the average ionization. Therefore, the presence of $\mathrm{Al}$ in the bow shock or of Ar in the jet does not introduce significant effects in the calculation of the properties of the bow shock, and in the following both CRSS models will not be considered.

For the Planck mean opacity and the RPL we obtain opposite behavior with respect to the average ionization, i.e., the largest values are obtained now for the CRSS models without including the radiation field, whereas the lowest ones are those provided by the SB model. The analysis of the monochromatic opacities and emissivities could help to explain this behavior. With this aim, we have represented them in Fig. 10 calculated at $300 \mathrm{~ns}$ and $z=15.0 \mathrm{~mm}$. In the representation of the monochromatic opacities, we have also included the Planck function since this is the weighting function in the calculation of the mean opacity.

From the figure we detect that in the energy photon range in which the Planck function presents a larger contribution (1-200 eV), the CRSS (100\% Ar) model obtains, in general, the largest values of the monochromatic opacity, overall in the photon energy range from 10 to $100 \mathrm{eV}$ where the Planck function reaches its largest values. On the other hand, the lowest values are obtained with the SB model and between them we found the results obtained with the CRSS model including the radiation field (CRSS+JRF) which is an expected result. The differences in the monochromatic opacities among the three models are related to the ions that contribute to the spectra. Thus, the CSDs of the CRSS and SB models present noticeable discrepancies, whereas the CSD of the CRSS+JRF is between them.

This behavior is also observed in the monochromatic emissivity. We have obtained that the main contributions to the RPL are the contributions to the emission spectra with photon energies up to $200 \mathrm{eV}$. We can observe from the figure that in the range of photon energies where the emission is more important (10-100 eV), the features in the spectrum obtained with the CRSS model without including radiation effects are more broadened than in the other two models and the depths of the valleys are lower as well. Since the RPL is obtained integrating the emissivity over the photon frequencies, these two facts could explain the behavior obtained with the different atomic kinetic models for this property.

We obtained similar conclusions in the analysis at $400 \mathrm{~ns}$ to those obtained at 300 ns. However, the relative differences in the properties studied were lower than at $300 \mathrm{~ns}$. This could be explained taking into account that at $400 \mathrm{~ns}$ the mass densities are, in general, larger than at $300 \mathrm{~ns}$, whereas the electron temperatures at both times are quite similar. Both facts lead to a diminution in the differences between CRSS and SB simulations.

As said before, the study carried out has been made using density and temperature profiles obtained in a hydrodynamic simulation assuming LTE regime for the plasma. From the numerical simulations it seems that, in the range of temperatures and densities analyzed, the CRSS model predicts that the plasma is more emissive than the SB model. This could imply that a NLTE radiation-hydrodynamic simulation would provide lower densities, temperatures, and velocities for the shock. A similar conclusion was obtained in a work in which radiation-hydrodynamic simulations using LTE and 

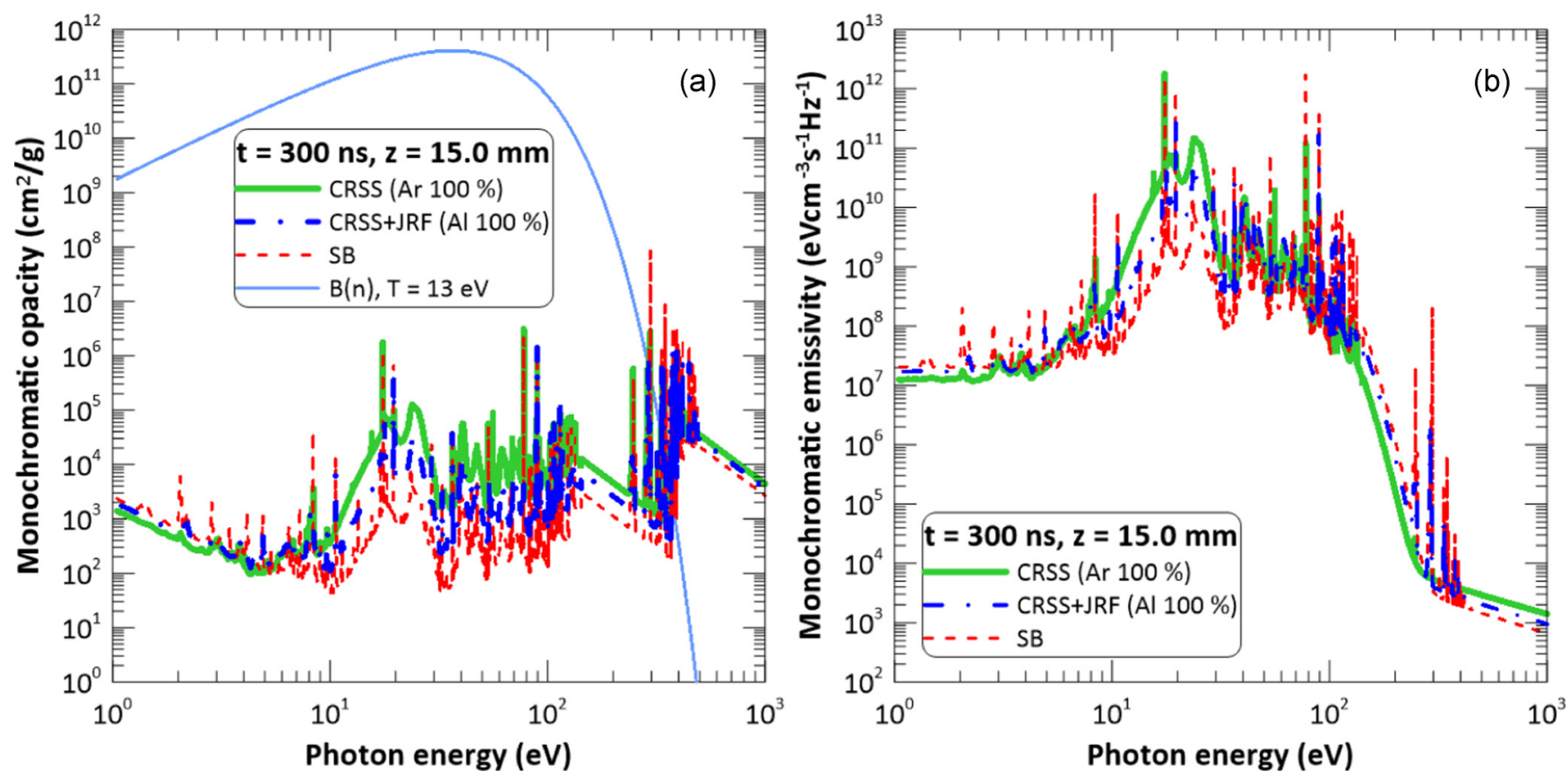

FIG. 10. Comparison of (a) the monochromatic opacities and (b) emissivities at $300 \mathrm{~ns}$ and $z=15.0$ mm calculated with the SB model and the CRSS models assuming the plasma as a monocomponent one with and without including the external radiation field and assuming the jet constituted only by Al.

NLTE models were made to analyze radiative shocks created in laboratory by laser-cluster interactions [55]. In any case, a radiation-hydrodynamic simulation coupled to our CRSS model would be necessary to confirm our assumption.

\section{Analysis of thermal instabilities in the bow shock}

Thermal instabilities are those that occur due to a balance between heating and cooling rates and they are of interest since they could be related to the formation of features in several astrophysics objects. These instabilities are expected to occur when the power of the heating source in the thermal energy equation is not adequate to ensure a steady temperature, and then cooling becomes the most important process [56-58]. Since the astrophysical phenomena can be reproduced currently in a scaled version, the study of thermal instabilities is also of interest in laboratory astrophysics. Thermal instabilities due to radiative cooling develop over the characteristic radiative cooling time which was given in Eq. (26).

Field [56] established the criteria for thermal instabilities assuming the unperturbed initial state as uniform and isothermal without velocity fields. These criteria were generalized for nonstationary mediums both for short- and long-wave perturbations by Shchenikov [58]. For the analysis we performed in this work we believed it more appropriate to consider the medium as nonstationary because this can be preheated by the radiation emitted from the shock, and followed the method developed by Shchenikov [58] (where a detailed explanation can be found), limiting ourselves to present in this section a brief explanation. The thermal instabilities can be classified according to a comparison between the length scale of the initial seeding perturbation and a characteristic scale of the medium, which is the sound crossing length $\lambda_{c}$, defined as

$$
\lambda_{c}=c_{s} t_{\mathrm{cool}}
$$

where $c_{s}$ is the ionic sound speed of the medium. For perturbations whose length is comparable to the sound crossing length, the pressure will be constant and they are isobaric instabilities [58]. In this case, the criterion for the instability is

$$
\frac{\rho_{u}}{T_{u}}\left(\frac{\partial L}{\partial \rho}\right)_{T_{u}}-\left(\frac{\partial L}{\partial T}\right)_{\rho_{u}}-\frac{L_{u}}{T_{u}}>0,
$$

where $L$ is the rate of heat loss (the cooling rate minus the heating rate) per gram and $u$ denotes the variables in the unperturbed medium.

When the length scales of the seeding perturbation are much greater than the sound crossing length, the instability has an effectively constant volume and is classified as isochoric and the criterion for the instability is given by [58]

$$
\left(\frac{\partial L}{\partial T}\right)_{\rho_{u}}-\frac{L_{u}}{T_{u}}<0 .
$$

Finally, if the length of the perturbation is considerably lower than the sound crossing length the developing instability is classified as adiabatic, and the condition that must be fulfilled for the thermal instability is

$$
\frac{1}{\gamma-1} \frac{\rho_{u}}{T_{u}}\left(\frac{\partial L}{\partial \rho}\right)_{T_{u}}+\left(\frac{\partial L}{\partial T}\right)_{\rho_{u}}-\frac{L_{u}}{T_{u}}<0 .
$$

As we can see from the equations, the criteria strongly depend on $L$ and its first derivatives with respect to density and temperature. In general, for radiative shocks, where the radiative cooling becomes a dominant process, the heat rate could be neglected in the calculation of $L$. In the experiment under analysis in this work there is no heating term and 
therefore $L$ represents the radiative cooling rate per gram. This one is obtained from the divergence of the radiative flux, which is given by Eq. (27). For optically thin plasmas, in which the opacity can be neglected, is given by the radiative power loss. For the range of plasma conditions under analysis we have obtained that the photon mean free paths, calculated with all the atomic kinetic models of this work, were always larger than the full widths at half maximum (FWHM) of the bow shock. Therefore, we concluded that the plasma in the bow shock can be considered as optically thin and then $L \approx \mathrm{RPL} / \rho$.

We have also analyzed the radiative character of the bow shock. For that purpose we compared the cooling time calculated using Eq. (26) in the optically thin approximation and the convective time calculated as the ratio between the FWHM of the bow shock and the sound speed in the medium, that was obtained taking the temperature just behind the front shock. The convective times obtained at 300 and $400 \mathrm{~ns}$ were around $100 \mathrm{~ns}-1 \mu \mathrm{s}$, respectively. On the other hand, the cooling times obtained using all the population kinetics models of this work were of the same order, around $1 \mathrm{ns,}$ which are considerably shorter than the convective times confirming the radiative character of the bow shock. This result agrees with the experimental observation of XUV emission [4] in the front of the bow shock which was almost as strong as at the tip of the jet, as mentioned in Sec. IV A.

Since both the cooling time and the criteria of thermal instabilities in the bow shock rely on the RPL we have studied the influence of the atomic description in the calculation of this property. In particular, we have made a comparison between the RPLs calculated in the DCA approach and the ones calculated in DLA approach including configuration interaction among levels belonging to the same nonrelativistic configuration. In the latter approach, the number of levels and transitions increase considerably with respect to the DCA approach. In Fig. 11, we have displayed the RPL calculated with both atomic approaches. From the figure we can observe a good agreement between both calculations, and therefore we can conclude that the DCA atomic approach is accurate enough to perform the analysis made in this work.

To analyze the possibility of the onset of thermal instabilities it is common to fit the RPL to a power law of the temperature and the mass density. We have locally fitted the RPL by the power law $C \rho^{\alpha} T^{\beta}$ using the PARPRA code [43] and imposing a maximum relative error of $0.1 \%$ in the fitting. The thermal instabilities criteria are then obtained in terms of the exponent of the temperature by substituting the power law of the RPL in $L$ into the Eqs. (29)-(31), and they are given by [59]

$$
\beta< \begin{cases}1 & \text { isochoric, } \\ \alpha & \text { isobaric, } \\ \frac{\gamma-\alpha}{\gamma-1} & \text { adiabatic }\end{cases}
$$

where $\gamma$ denotes the adiabatic index. The fitting of the RPL and these proposed criteria have been already used to predict the possibility of thermal instabilities in experiments of convergent radiative shocks in argon and neon produced in a cylindrical liner Z-pinch configuration [24] and also in experiments of blast waves launched in clusters of xenon $[17,26,60]$. In both cases, the results obtained theoretically were consistent with the experimental observations.

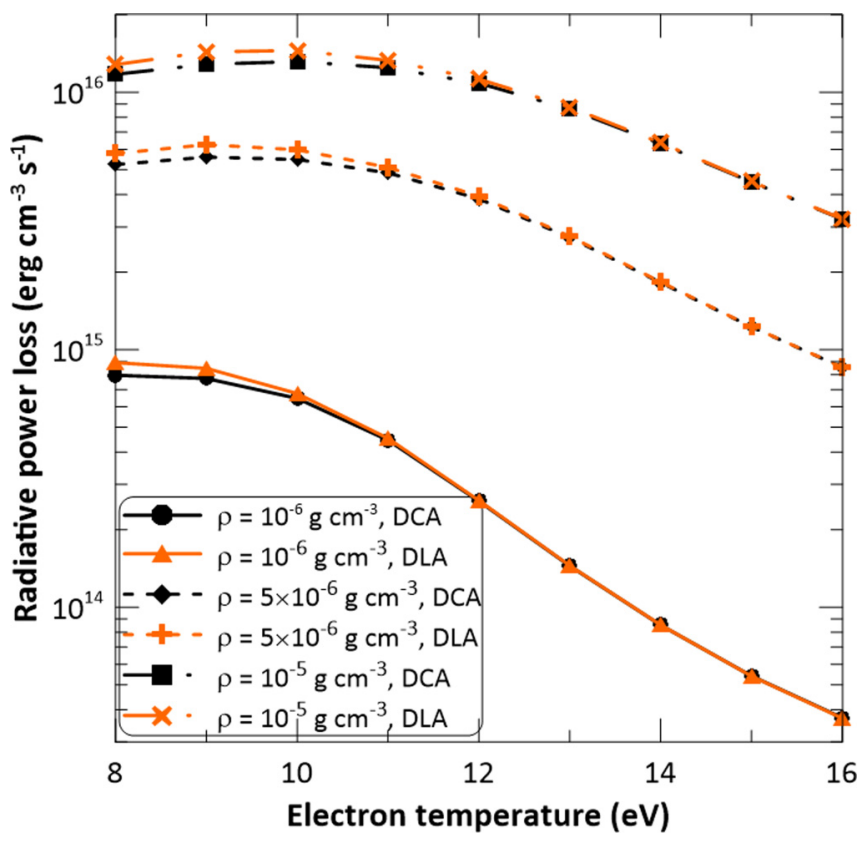

FIG. 11. Comparison of the radiative power loss calculated under two atomic approaches, DCA and DLA.

In Fig. 12, we have displayed the exponent $\beta$ and the three criteria of Eq. (32) calculated using the different atomic kinetic models analyzed in this work as a function of the electron temperature. We have chosen the intermediate value of the density of matter for the representation since we have detected that both the criteria and the value of $\beta$ do not change appreciably with the density. We can observe that the SB model predicts the possibility of thermal instabilities in the whole range of electron temperatures. With respect to the CRSS models, the ones that included the radiation field from the jet predict that the isochoric and isobaric instabilities can occur at any temperature of the range and for the adiabatic one only the range of temperatures $8-10 \mathrm{eV}$ is not allowed. For the optically thin CRSS model, i.e., without including the external radiation field due to the jet, similar results are obtained for the adiabatic instability, although the range where it is not allowed is reduced to $8-9 \mathrm{eV}$, and in this case there is a small range of temperatures, around $8-8.5 \mathrm{eV}$, where the criterion for the isochoric instability is not fulfilled. Therefore, from the analysis we conclude that all the kinetic models predict the possibility of isobaric instabilities in the bow shock.

In the experiment under analysis in this work the images obtained using a dark-field laser Schlieren diagnostic showed the presence of small-scale structures $(<100 \mu \mathrm{m})$ forming on the shock [4]. Since the bow shock is strongly radiatively cooled, its fragmentation could be related to a local thermal isobaric instability that could produce a condensation by radiative cooling.

As said before, for isobaric instability to occur, the length of the perturbation should be comparable to the sound crossing length given by Eq. (29). In Fig. 13, we have represented the sound crossing length as a function of the temperature and for three densities of the range of the experiment. The calculations were made with the SB and CRSS optically thin models and with the CRSS one including the external radiation at two 

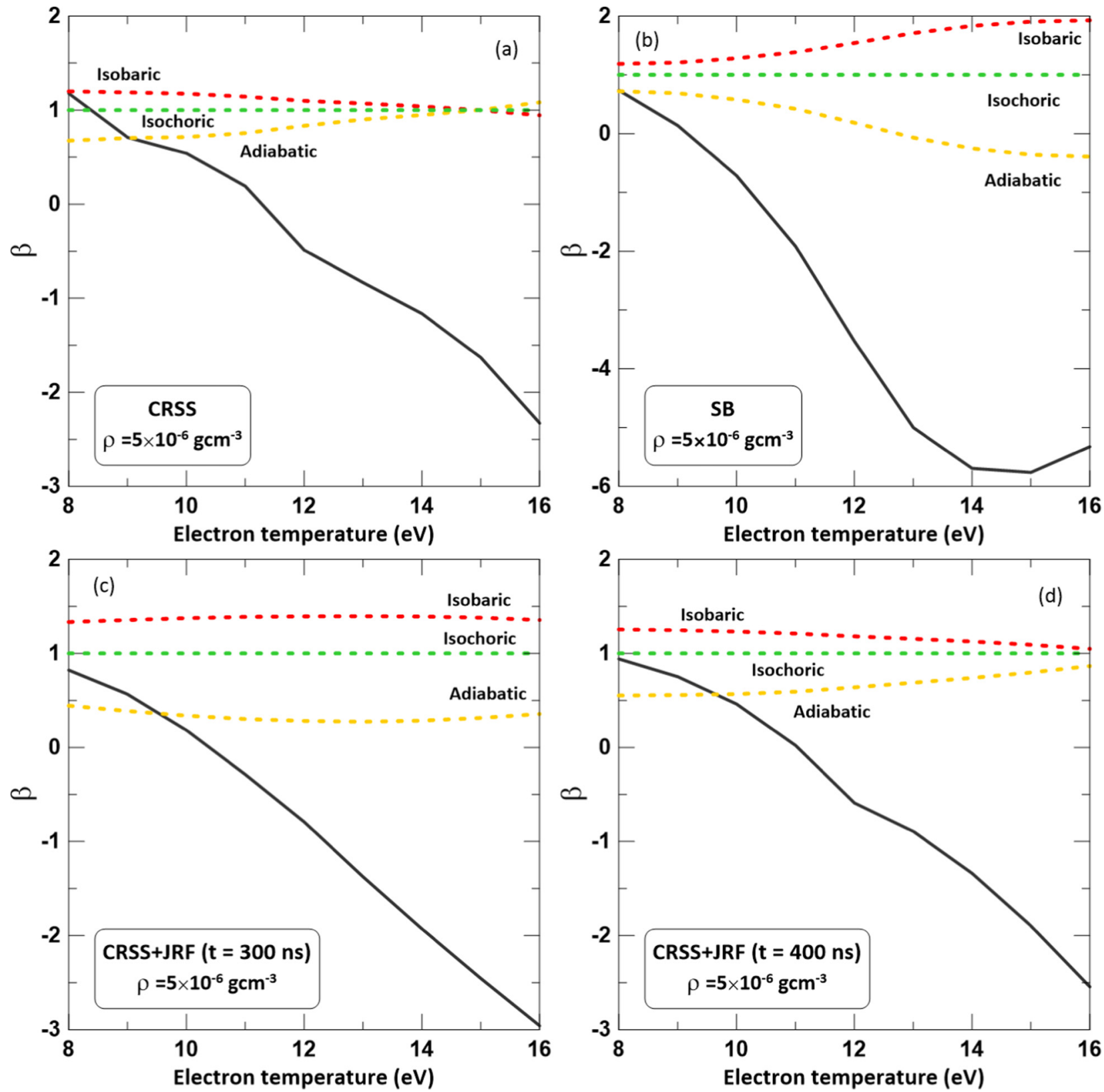

FIG. 12. Exponent of the temperature $\beta$ and the three criteria for thermal instabilities as a function of the temperature.

times, 300 and $400 \mathrm{~ns}$, since the radiation emitted by the jet is not the same at each time being less intense at later times. From the figure we can detect that the largest values of sound crossing lengths are obtained with the SB model, whereas the lowest ones are those provided by the optically thin CRSS model. This fact is due to the cooling times for the latter model are the lower ones which is expected since they depend on the RPLs [see Eq. (26)] and as was shown in Sec. IV B, the RPLs obtained with this model were the largest ones. The behavior of the RPL also explains why the values of $\lambda_{c}$ obtained using the CRSS model including the radiation field are between the optically thin CRSS and SB models. Furthermore, since the radiation field is less intense at $400 \mathrm{~ns}$, the sound crossing lengths are, in this case, closer to the ones obtained in the optically thin case.
The figure also shows that the sound crossing length increases with the temperature and this can be explained by the subsequent increase of the speed of the sound and cooling times. We also observe that, in general, $\lambda_{c}$ increases as the density of matter decreases. In this case, this behavior is ruled by the cooling time given by Eq. (26). In the range of densities analyzed, when the mass density changes in one order of magnitude (from $10^{-6}$ to $10^{-5} \mathrm{~g} \mathrm{~cm}^{-3}$ ), the RPL changes in two orders of magnitude and this is the reason of the decrease in the cooling time with the density. Under some conditions of electron temperature and density of matter, the average ionization also plays a role in the cooling time and this explains the discrepancies in the general behavior detected at the two highest temperatures in the optically thin CRSS model and at the low temperature range in the SB model. 

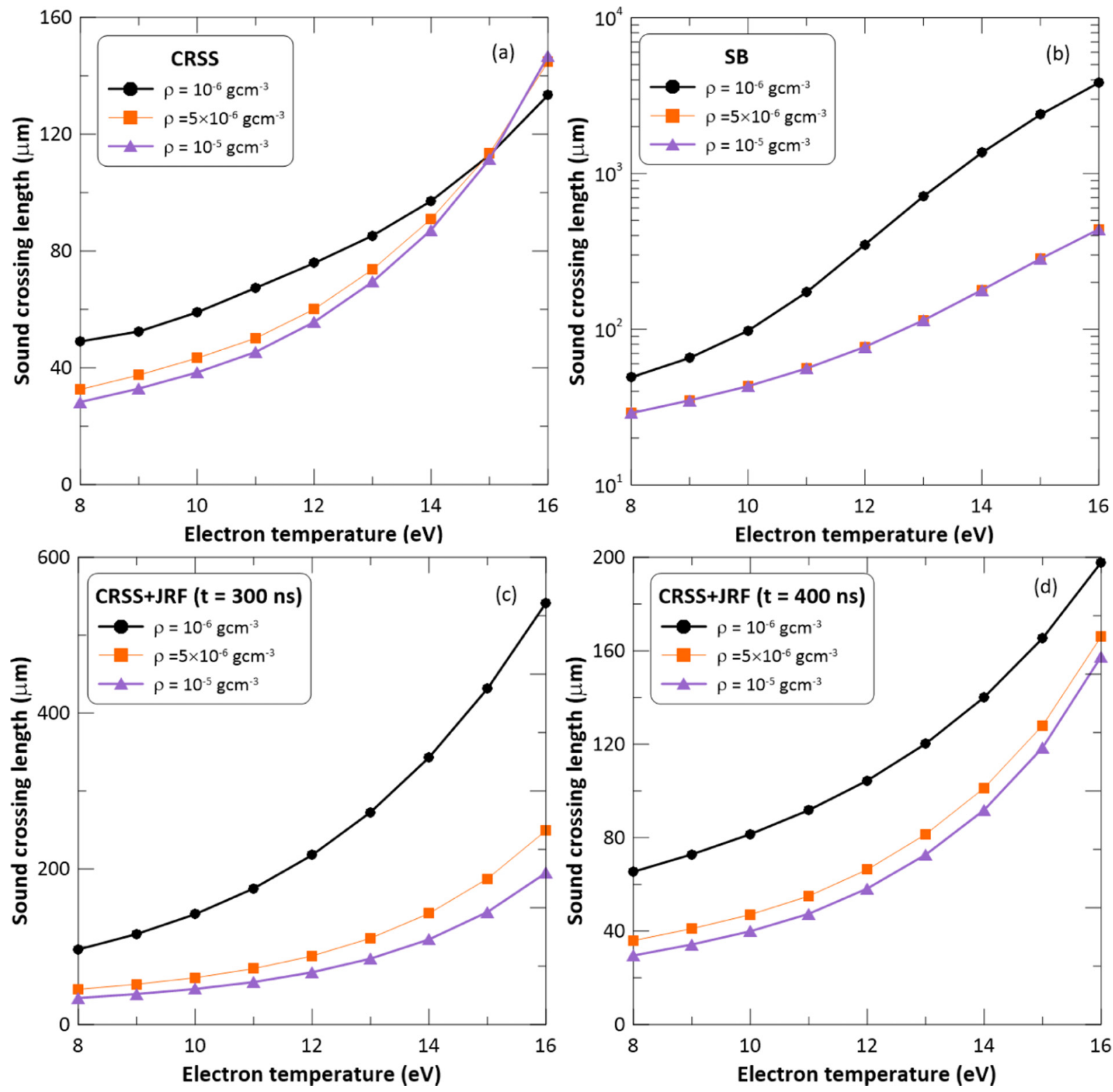

FIG. 13. Sound crossing length calculated using three population kinetic models as a function of the temperature and for three mass densities.

In the experiment, the small structures are observed at $400 \mathrm{~ns}$, where the range of temperatures in the bow shock is between 10 to $14 \mathrm{eV}$ and the range of densities the one shown in the figure. According to Fig. 13, the lengths of the structures observed in the experiment are comparable to the sound crossing lengths obtained using CRSS and the CRSS+JRF ( $t=400 \mathrm{~ns}$ ) models for the whole range of densities. On the other hand, this criterion is not fulfilled by the SB model at the lowest density and for the intermediate density at the highest temperature. According to the analysis carried out in Sec. IV B, it is clear that the plasma is not in LTE and we conclude in this analysis that its use leads both to inaccurate values of the plasma microscopic properties and to incorrect predictions about the possibility of the onset of isobaric thermal instabilities in the bow shock.
The sound crossing length sets an upper limit for the scale of isobaric condensation. However, there is also a lower limit since thermal conduction can suppress short wavelength perturbations [56]. This lower limit is the Field's length, given by

$$
\lambda_{F}=2 \pi\left[(\gamma-1) \chi_{\mathrm{th}} t_{\mathrm{cool}}\right]^{1 / 2},
$$

where $\chi_{\mathrm{th}}$ is the thermal diffusivity and $\gamma$ is the polytropic index. We calculated the Fields's length for the range of plasma conditions of the experiment using the CRSS models and we obtained that for the whole range of conditions this length was around $1 \mu \mathrm{m}$ that is considerably lower than the length of the structures detected and then the thermal conduction would not suppress the instability.

Therefore, from our analysis we can conclude that the small structures observed experimentally could be related 
with isobaric instabilities due to radiative cooling and that a correct theoretical prediction of them requires a NLTE atomic kinetic model.

\section{CONCLUSIONS}

In this work, we have studied the accuracy of LTE assumption in the calculation of several microscopic properties of argon plasmas in ranges of mass densities $\left(10^{-6}-10^{-1} \mathrm{~g} \mathrm{~cm}^{-3}\right)$ and electron temperatures $1-100 \mathrm{eV}$ typically found in laboratory astrophysical experiments on radiative shocks. For this purpose, we have made comparisons with simulations carried out with a CRSS model whose accuracy for the simulation of microscopic properties of argon plasmas was previously checked. We have obtained that for the densities between $10^{-1}$ and $10^{-2} \mathrm{~g} \mathrm{~cm}^{-3}$, the LTE assumption is accurate to calculate the plasma radiative properties although for the latter some appreciable differences are detected (around 30\%) for temperatures higher than $60 \mathrm{eV}$. For mass densities lower than $10^{-2} \mathrm{~g} \mathrm{~cm}^{-3}$, the discrepancies between both simulations are significant.

Furthermore, we have made an analysis of the influence of the atomic kinetic model used to calculate plasma microscopic properties of a bow shock propagating in argon generated in a particular HED laboratory plasma astrophysics experiment. The microscopic properties studied were the average ionization, the charge state distributions, the monochromatic opacities and emissivities, the Planck mean opacity, and the radiative power loss. All of them are properties of interest in radiative-hydrodynamics simulations and the radiative power loss, in particular, in the theoretical prediction of the onset of thermal instabilities by radiative cooling in the postshock region. The range of mass densities and electron temperatures of the experiment were extracted from a hydrodynamic simulation using the GORGON code that assumed LTE regime for the plasma. The density and temperature profiles were given in the jet axial direction at two times, 300 and 400 ns. For the study carried out in this work, we have considered population kinetic models either assuming the plasma in LTE or NLTE. For the LTE situation, we have solved the Saha-Boltzmann equations. For the NLTE regime, we have solved the rate equations in stationary state and we have considered different situations for the plasma in the bow shock: pure argon plasma, a mixture of aluminum and argon, and the inclusion or not of the axial radiation field coming from the jet as an external field. The accuracy of the CRSS model was checked by comparisons with other codes. From the analysis of the microscopic properties we obtained that the largest differences for all the properties arise between the SB and the CRSS optically thin models. The results obtained using the CRSS models including the external radiation field are located between them. The differences among the codes are greater at $300 \mathrm{~ns}$ than at $400 \mathrm{~ns}$ since at later times the density is slightly higher than at $300 \mathrm{~ns}$, which favors the LTE. We have observed that including a little amount of $\mathrm{Al}(5 \%)$ in the bow shock does not introduce significant modifications in the microscopic properties with respect to the pure Ar situation. This conclusion is also obtained when the external radiation is modeled assuming the jet as pure $\mathrm{Al}$ or a mixture of $\mathrm{Ar}$ and $\mathrm{Al}$ (50\% each). From the analysis we concluded that for the plasma conditions considered, the LTE assumption is inaccurate for the calculation of the microscopic plasma properties. On the other hand, it seems that the external radiation field should be considered in the simulations since it introduces modifications with respect to the optically thin situation.

In the experiment, small structures in the bow shock were observed at $400 \mathrm{~ns}$ that could be related with condensations due to isobaric thermal instabilities. In this work, we have made a theoretical analysis of the possibility of the onset of thermal instabilities due to the cooling rate in the range of plasma conditions of the experiment. First, we concluded that the plasma was optically thin and radiative. Therefore, the analysis of thermal instabilities relies on the RPL. We checked that for that property the DCA atomic approach was accurate enough. The fitting by a power law of the RPL provided us the criteria for the three types of thermal instabilities and the plasma conditions in which they can arise. This was made using the SB and the CRSS with or without external field models. Beyond the fulfillment of the criterion of the isobaric instability, the length scale of the isobaric condensation should be of the same order than the sound crossing length. The simulations made with both CRSS models verified these two conditions, but not the LTE model. From our study we conclude that the small structures experimentally observed could be due to an isobaric instability due to the radiative cooling of the bow shock.

The study carried out in this work was made as a post-processing of a hydrodynamic simulation. A subsequent study would imply to introduce the CRSS models in the hydrodynamic simulation and then to analyze the changes that they could introduce in the macroscopic simulation. Besides, in future experiments we could be able to measure some of the plasma properties analyzed in this work using some of the laser-driven diagnostics systems of MAGPIE, as for example the laser interferometric imaging for the plasma electron density and the collective Thomson scattering whose spectral shape is sensitive to the ion temperature and the product of the average ionization and the electron temperature (see [61] for a detailed explanation). This could enable us to perform comparisons with our theoretical simulations.

\section{ACKNOWLEDGMENTS}

This work has been supported by the EUROfusion Consortium TASK AGREEMENT WPENR: Enabling Research IFE, Project No. AWP15-ENR-01/CEA-02 and by the Project of the Spanish Government with reference FIS2016-81019-P.
[1] P. Keiter, K. Mussack, and S. Kleina, High Energy Density Phys. 9, 319 (2013).

[2] G. Rochau, J. Bailey, R. Falcon, G. Loisel, T. Nagayama, R.. Mancini, D. Hall, I.and Winget, M. Montgomery, and D. Liedahl, Phys. Plasmas 21, 056308 (2014).
[3] M. Le Pennec, X. Ribeyre, J. Ducret, and S. Turck-Chieze, High Energy Density Phys. 17, 162 (2015).

[4] F. Suzuki-Vidal, M. Bochhi, S. Lebedev, G. Swandling, G. Burdiak, S. Bland, P. de Grouchy, G. Hall, E. Harvey-Thomsom, E. Khoory, S. Pantakar, L. Pickworth, J. Skidmore, R. Smith, 
J. Chittenden, M. Krishnan, R. Maden, K. Wilson-Elliot, A. Ciardi, and A. Frank, Phys. Plasmas 19, 022708 (2012).

[5] M. Manuel, C. Kuranz, A. Rasmus, S. Klein, M. MacDonald, M. Trantham, J. Fein, P. Belancourt, R. Young, P. Keiter, R. Drake, B. Pollock, J. Park, A. Hazi, G. Williams, and H. Chen, High Energy Density Phys. 17, 52 (2015).

[6] M. Bennett, S. Lebedev, G. Hall, L. Suttle, G. Burdiak, F. Suzuki-Vidal, J. Hare, G. Swadling, S. Patankar, M. Bocchi, J. Chittenden, R. Smith, A. Frank, E. Blackman, R. Drake, and A. Ciardi, High Energy Density Phys. 17, 63 (2015).

[7] D. Ryutov, R. Drake, J. Kane, E. Liang, B. Remington, and W. Wood-Vasey, Astrophys. J. 518, 821 (1999).

[8] D. Ryutov, B. Remington, H. Robey, and R. Drake, Phys. Plasmas 8, 1804 (2000).

[9] B. A. Remington, R. P. Drake, and D. D. Ryutov, Rev. Mod. Phys. 78, 755 (2006).

[10] J. Castor, Astrophys. Space Sci. 307, 207 (2007).

[11] E. Falize, D. Bouquet, and C. Michaut, Astrophys. Space Sci. 322, 107 (2009).

[12] S. Bouquet, E. Falize, C. Michaut, C. Gregory, B. Loupias, T. Vinci, and M. Koenig, High Energy Density Phys. 6, 368 (2010).

[13] B. Fryxell, R. Drake, C. Kuranz, and E. Myre, Phys. Scr. T155, 014017 (2013).

[14] C. Krauland, R. Drake, C. Kuranz, R. Sweeney, M. Grosskopf, S. Klein, R. Gillespie, P. Keiter, B. Loupias, and E. Falize, Phys. Plasmas 20, 056502 (2013).

[15] U. Chaulagain, C. Stehle, J. Larour, M. Kozlova, F. SuzukiVidal, P. Barroso, M. Cotelo, P. Velarde, R. Rodriguez, J. Gil, A. Ciardi, O. Acef, J. Nejdl, L. de Sa, R. Singh, L. Ibgui, and N. Champion, High Energy Density Phys. 17, 106 (2015).

[16] J. Osterhoff, D. Symes, A. Edens, A. Moore, E. Hellewell, and T. Ditmire, New J. Phys. 11, 023022 (2009).

[17] D. Symes, H. Hohenberger, H. Doyle, R. Smith, A. Moore, E. Gumbrell, R. Rodriguez, and J. Gil, Nucl. Instrum. Methods Phys. Res., Sect. A 653, 186 (2011).

[18] F. Suzuki-Vidal, S. Lebedev, S. Bland, G. Hall, G. Swadling, A. Harvey-Thompson, G. Burdiak, P. de Grouchy, J. Chittenden, A. Marocchino, M. Bocchi, A. Ciardi, A. Frank, and S. Bott, Astrophys. Space Sci. 336, 41 (2011).

[19] F. Suzuki-Vidal, S. Lebedev, M. Krishnan, J. Skidmore, G. Swadling, M. Bocchi, A. Harvey-Thompson, S. Patankar, G. Burdiak, P. de Grouchy, L. Pickworth, S. Stafford, L. Suttle, M. Bennett, S. Bland, J. Chittenden, G. Hall, E. Khoory, R. Smith, A. Ciardi, A. Frank, R. Madden, K. Wilson-Elliot, and P. Coleman, High Energy Density Phys. 9, 141 (2013).

[20] B. Albertazzi, A. Ciardi, M. Nakatsutsumi, T. Vinci, J. Beard, R. Bonito, J. Billette, M. Borghesi, Z. Burkley, S. Chen, T. E. Cowan, T. Herrmannsdorfer, D. P. Higginson, S. A. Kroll, F. Pikuz, K. Naughton, L. Romagnani, C. Riconda, G. Revet, R. Riquier, H. Schlenvoigt, I. Y. Skobelev, A. Y. Faenov, A. Soloviev, M. Huarte-Espinosa, A. Frank, O. Portugall, H. Pepin, and J. Fuchs, Science 346, 325 (2014).

[21] F. Suzuki-Vidal, S. Lebedev, M. Bocchi, M. Krishnan, G. Swadling, G. Burdiak, S. Bland, P. de Grouchy, G. Hall, A. Harvey-Thompson, E. Khoory, L. Pickworth, J. Skidmore, J. Chittenden, K. Wilson-Elliot, R. Madden, and A. Ciardi, Shock Waves 22, 249 (2012).

[22] G. Burdiak, S. Lebedev, R. Drake, A. Harvey-Thompson, G. Swadling, F. Suzuki-Vidal, J. Skidmore, L. Suttle, E. Khoory,
L. Pickworth, P. de Grouchy, G. Hall, S. Bland, M. Weinwurm, and J. Chittenden, High Energy Density Phys. 9, 52 (2013).

[23] G. Burdiak, S. Lebedev, A. Harvey-Thompson, G. Swadling, F. Suzuki-Vidal, G. Hall, E. Khoory, L. Pickworth, S. Bland, P. de Grouchy, J. Skidmore, L. Suttle, M. Bennett, N. Niasse, R. Williams, K. Blesener, L. Atoyan, A. Cahill, C. Hoyt, W. Potter, E. Rosenberg, P. Schrafel, and B. Kusse, Phys. Plasmas 21, 033302 (2014).

[24] G. Burdiak, S. Lebedev, F. Suzuki-Vidal, G. Swadling, S. Bland, N. Niasse, L. Suttle, M. Bennet, J. Hare, M. Weinwurm, R. Rodríguez, J. Gil, and G. Espinosa, J. Plasma Phys. 81, 365810301 (2015).

[25] P. Nicolai, C. Stenz, V. Tikhonchuk, X. Ribeyre, A. Kasperczuk, T. Pisarczyk, L. Juha, E. Krousky, K. Masek, M. Pfeifer, K. Rohlena, J. Skala, J. Ullschmied, M. Kalal, D. Klir, J. Kravarik, P. Kubes, and P. Pisarczyk, Astrophys. Space Sci. 322, 11 (2009).

[26] M. Hohenberger, D. R. Symes, J. Lazarus, H. W. Doyle, R. E. Carley, A. S. Moore, E. T. Gumbrell, M. M. Notley, R. J. Clarke, M. Dunne, and R. A. Smith, Phys. Rev. Lett. 105, 205003 (2010).

[27] A. J. Visco, R. P. Drake, S. H. Glenzer, T. Doppner, G. Gregori, D. H. Froula, and M. J. Grosskopf, Phys. Rev. Lett. 108, 145001 (2012).

[28] S. Olsson Robbie, H. Doyle, D. Symes, and R. Smith, High Energy Density Phys. 8, 55 (2012).

[29] G. Yamada, H. Kawazoe, and S. Obayashi, J. Fluid Sci. Technol. 11, JFST0005 (2016).

[30] R. Singha, C. Stehle, F. Suzuki-Vidal, M. Kozlovac, J. Larourd, U. Chaulagain, T. Clayson, J. Nejdl, M. Krusc, J. Dostale, R. Dudzake, P. Barroso, O. Acef, V. P. Cotelo, M. and, R. Rodriguez, and J. Gil, High Energy Density Phys. (to be published).

[31] P. J. Wiita, Nature 355, 499 (1992).

[32] J. Chittenden, S. Lebedev, B. Oliver, E. Yu, and M. Cuneo, Phys. Plasmas 11, 1118 (2004).

[33] A. Ciardi, S. Lebedev, A. Frank, E. Blackman, J. Chittenden, C. Jennings, D. Ampleford, S. Bland, S. Bott, J. Rapley, G. Hall, F. Suzuki-Vidal, A. Marocchino, T. Lery, and C. Stehle, Phys. Plasmas 14, 056501 (2007).

[34] H. Scott and S. Hansen, High Energy Density Phys. 6, 39 (2010).

[35] M. F. Gu, Can. J. Phys. 86, 675 (2008).

[36] J. Bauche, C. Bauche-Arnoult, and M. Klapisch, Adv. At. Mol. Phys. 23, 131 (1987).

[37] J. C. Stewart and K. D. Pyatt, Astrophys. J. 144, 1203 (1966).

[38] W. Lotz, Z. Phys. 216, 241 (1968).

[39] H. Van Regemorter, Astrophys. J. 136, 906 (1962).

[40] H. A. Kramers, Philos. Mag. 46, 836 (1923).

[41] H. R. Griem, Principles of Plasma Spectroscopy (Cambridge University Press, Cambridge, UK, 1997).

[42] M. Klapisch and M. Busquet, New J. Phys. 15, 015012 (2013).

[43] R. Rodríguez, G. Espinosa, J. Gil, J. Rubiano, M. Mendoza, P. Martel, and E. Mínguez, Commun. Comput. Phys. 16, 612 (2014).

[44] Y. Li, C. Gao, Q. Tian, J. Zeng, and J. Yuan, Phys. Plasmas 22, 113302 (2015).

[45] R. Florido, R. Rodríguez, J. M. Gil, J. G. Rubiano, P. Martel, E. Mínguez, and R. C. Mancini, Phys. Rev. E 80, 056402 (2009). 
[46] R. Rodríguez, R. Florido, J. M. Gil, J. G. Rubiano, D. Suárez, P. Martel, E. Mínguez, and R. C. Mancini, Commun. Comput. Phys. 8, 185 (2010).

[47] R. Rodríguez, R. Florido, J. M. Gil, J. G. Rubiano, P. Martel, and E. Mínguez, Laser Part. Beams 26, 433 (2008).

[48] D. Mihalas, Stellar Atmospheres (W. H. Freeman, San Francisco, 1970).

[49] M. S. Dimitrijevic and N. Konjevic, Astron. Astrophys. 172, 345 (1987).

[50] S. J. Rose, J. Phys. B: At., Mol. Opt. Phys. 25, 1667 (1992).

[51] R. J. Rutten, Radiative Transfer in Stellar Atmospheres (Sterrekundig Instituut, Utrecht, 1995).

[52] F. Serduke, E. Mínguez, S. Davidson, and C. Iglesias, J. Quant. Spectrosc. Radiat. Transfer 65, 527 (2000).

[53] C. Fontes, J. J. Abdallah, C. Bowen, R. Lee, and Y. Ralchenko, High Energy Density Phys. 5, 15 (2009).

[54] G. Espinosa, J. Gil, R. Rodriguez, J. Rubiano, M. Mendoza, P. Martel, E. Minguez, F. Suzuki-Vidal, S. Lebedev, G. Swadling, G. Burdiak, L. Pickworth, and J. Skidmore, High Energy Density Phys. 17, 74 (2015).
[55] R. Scott, N. Booth, S. Hawkes, D. Symes, H. Doyle, S. OlssonRobbie, H. Lowe, C. Price, D. Bigourd, S. Patankar, K. Mecseki, R. A. Smith, J. Fyrth, and E. Gumbrell, Modelling radiative shocks created by Central Laser Facility Annual Report, 2013-2014, https://www.clf.stfc.ac.uk/Pages/CLF_Annual_ Report_2ndJuneproof-B.pdf.

[56] G. Field, Astrophys. J. 142, 531 (1965).

[57] J. J. Hunter, Astrophys. J. 161, 451 (1970).

[58] Yu. A. Shchekinov, Sov. Astron. 22, 182 (1978) [Astron. Zh. 55, 311 (1978)].

[59] R. Rodriguez, G. Espinosa, J. M. Gil, C. Stehle, F. Suzuki-Vidal, J. G. Rubiano, P. Martel, and E. Minguez, Phys. Rev. E 91, 053106 (2015).

[60] R. Rodríguez, J. M. Gil, G. Espinosa, R. Florido, J. Rubiano, M. A. Mendoza, P. Martel, E. Mínguez, D. Symes, M. Hohenberger, and R. Smith, Plasma Phys. Controlled Fusion 54, 045012 (2012).

[61] G. Swadling, S. Lebedev, G. Hall, S. Patankar, N. Stewart, R. Smith, A. Harvey-Thompson, G. Burdiak, P. de Grouchy, J. Skidmore, L. Suttle, F. Suzuki-Vidal, S. Bland, K. Kwek, L. Pickworth, M. Benneett, J. Hare, W. Rozmus, and J. Yuan, Rev. Sci. Instrum. 85, 11E502 (2014). 